\title{
Energetics of transient-eddy and inter-member variabilities in global and regional climate model simulations
}

\author{
Oumarou Nikiéma ${ }^{1}$ D $\cdot$ René Laprise $^{1} \cdot$ Bernard Dugas $^{1}$
}

Received: 19 April 2017 / Accepted: 15 September 2017 / Published online: 13 October 2017

(c) The Author(s) 2017. This article is an open access publication

\begin{abstract}
Available Enthalpy (AE) and Kinetic Energy (KE) associated with the natural Transient-Eddy (or TimeVariability, TV) and models' Internal Variability (or Intermember Variability, IV) are studied using two ensembles of simulations, one from a nested Regional Climate Model (RCM) driven by reanalyses over a regional domain covering eastern North America, and one from a Global Climate Model (GCM), and the Era-interim reanalyses as reference. The fields of TV and IV energies are first examined, both globally and over the regional domain. Results from GCM simulations reveal that GCM TV is similar to that of reanalyses, confirming the realism of the GCM simulations, and TV and IV are approximately equal, in agreement with the ergodicity property. For RCM simulations, TV energies are similar to those of reanalyses driving them. On the other hand, the IV energies of reanalyses-driven RCM simulations are much smaller than those of the GCM, because of the control exerted by the lateral boundary conditions imposed in nested models. While GCM IV energies present similar seasonal variations as the TV energies, the RCM IV greatly fluctuates in time, with short episodes of large variations. The second part of this study is devoted to the analysis of TV and IV energetic budgets. Results indicate similar physical interpretations of conversions, generations and destructions for both TV and IV energetics, although TV is
\end{abstract}

Electronic supplementary material The online version of this article (doi:10.1007/s00382-017-3918-0) contains supplementary material, which is available to authorized users.

Oumarou Nikiéma

oumarou.nikiema@uqam.ca

1 ESCER Centre, Département des Sciences de la Terre et de l'Atmosphère, Université du Québec à Montréal (UQAM), Montréal, Canada associated with natural phenomena of weather disturbances and IV is a model feature contributing to the uncertainties of simulations.

Keywords Ensemble of climate simulations - Transient eddies · Internal variability $\cdot$ Available enthalpy $\cdot$ Kinetic energy $\cdot$ Atmospheric energy conversions

\section{Introduction}

Climate models are powerful tools that allow better understanding of past, present and future climatic phenomena. One advantage that climate models provide is their ability to be used in sensitivity experiments using ensembles of simulations. As first demonstrated by Lorenz (1963) for nonlinear systems, climate models exhibit different trajectories in phase space due to their sensitivity to initial conditions (IC).

The study of atmospheric variables using climate models simulations indicates different forms of variability, notably the natural Transient-Eddy (or Time Variability-TV), and Internal (or Inter-member) Variability (IV) when ensembles of simulations are considered. The first form of variability (TV) has a physical meaning since it reflects the passage of weather events (e.g. storms, cyclones, floods, etc.), while the second one (IV) is specific to models and it represents a measure of model's uncertainties.

Regional Climate Models (RCM) are used to make climate simulations at local scales, but they need to be driven by Lateral Boundary Conditions (LBC) data from reanalysis or low-resolution Global Climate Models (GCM) simulations. Over the last two decades, several studies have paid particular attention on RCMs' IV, e.g. Giorgi and Bi (2000), Weisse et al. (2000) Rinke and Dethloff (2000), Christensen 
et al. (2001), Caya and Biner (2004), Rinke et al. (2004), Lucas-Picher et al. (2004), Alexandru et al. (2007), de Elía et al. (2008) and Lucas-Picher et al. (2008), to name but a few. Recently, Nikiéma and Laprise (2011a, b) have developed a diagnostic approach that furthers our physical understanding of RCM's IV as a source of uncertainty. They established IV diagnostic equations for different atmospheric variables taking into account the model equations and Reynolds' decomposition applied to the atmospheric field equations, as well as some statistic tools since an ensemble of simulations is considered. Their results suggested a close parallel between the energy conversions associated with the time fluctuations of IV and the TV energy conversions taking place in weather systems as first identified by Lorenz (1955, 1967).

The temperature and the wind are two important dynamical atmospheric variables, and they allow defining Potential Energy (PE) and Kinetic Energy (KE). Nowadays, the atmospheric global circulation is well understood following the energy cycle mechanism proposed by Lorenz (1955). He introduced the concept of Available Potential Energy (APE), which is transformed into KE through energy conversions. Indeed, to better understand the role of weather systems in the atmospheric energetics, Lorenz further decomposed the energy fields into components associated with the zonalmean atmospheric state and departures thereof, termed eddies. Much of our current understanding of global atmospheric energetics derives from Lorenz' seminal work (e.g. Oort 1964a, b; van Mieghem 1973; Newell et al. 1972, 1974; Boer 1974; Pearce 1978); Pexioto and Oort 1992; to name just a few). Following an alternative approach, Marquet (1991, 2003a, b) proposed a formalism based on Available Enthalpy (AE) instead of APE. Inspired by these previous works, Nikiéma and Laprise $(2013,2015)$ established an approximate energy cycle of IV applicable for a limited-area domain, and afterward adapted to study TV in a particular intense storm observed over the North America (Clément et al. 2016). Their methodology used a decomposition of atmospheric variables into their time-mean state and time variability (perturbations) rather than into zonal-mean and deviations as it is often done for global energetics studies (Lorenz 1955, 1967).

The present study is an extension of previous work with the main goal to compared and analyse AE and KE energies associated with the natural TV and models IV, at global and regional scales. This study is done using three datasets, from ensembles of GCM and RCM simulations, as well as Era-Interim reanalysis considered as reference. Two sets of 50- and 30-member of simulations were performed using the nested fifth-generation Canadian RCM (CRCM5) and it global version (called GEM-Global), respectively. The CRCM5's simulations were run over a North America regional domain and it was driven by the same $\mathrm{LBC}$ from
Era-Interim. For both climate models, all the members were run over the same period and the difference between members is only the initial data used to start (i.e. IC) each simulation. For two consecutive runs, the ICs are the Era-Interim data shifted by $24 \mathrm{~h}$ and models output are archived over the same period of interest. The paper is organised as follows: the following Sect. 2 describes the methodology and data used; thereafter, results are presented and discussed in Sect. 3. TV and IV energies are analysed at global and regional scales, as well as energy budgets. Finally, the conclusion is summarised in Sect. 4.

\section{Evaluation methods and data}

\subsection{Available enthalpy and kinetic energy associated with TV and IV}

In an ensemble of $N$-member simulations, each atmospheric variable $\varphi_{n}$, where $n$ represents the simulation's index, can be split in two parts: the ensemble-mean noted $\langle\varphi\rangle$ and the member deviation thereof noted $\varphi_{n}^{\prime}=\varphi_{n}-\langle\varphi\rangle$. In the same way for each simulation $n$, the time deviation over a period of interest can be written as $\varphi_{n}^{\prime \prime}=\varphi_{n}-\overline{\varphi_{n}}$, where $\bar{\varphi}_{n}$ and $\varphi_{n}^{\prime \prime}$ design the time-mean and deviation thereof, respectively.

Following Nikiéma and Laprise (2011a, b), the IV for any atmospheric variable $\varphi_{n}$ can be evaluated by calculating the inter-member variance $\sigma_{\varphi}^{2}$ as:

$\sigma_{\varphi}^{2}(i, j, k, t) \approx \frac{1}{N} \sum_{n=1}^{N} \varphi_{n}^{\prime 2}(i, j, k, t) \equiv\left\langle\varphi_{n}^{\prime 2}\right\rangle$

The TV can similarly be evaluated as:

$\sum_{\varphi_{n}}^{2}(i, j, k) \approx \frac{1}{\tau} \sum_{t=1}^{\tau} \varphi_{n}^{\prime \prime 2}(i, j, k, t) \equiv \varphi_{n}^{\prime \prime 2}$

where $\tau$ is the number of archive intervals over the period of interest. It is noteworthy that IV is a function of time, unlike $\mathrm{TV}$; hence the time-mean of $\operatorname{IV}\left(\overline{\left\langle\sigma_{\varphi}^{2}\right\rangle} \equiv \overline{\left\langle\varphi_{n}^{\prime 2}\right\rangle}\right)$ will be considered in order to compare with TV.

The temperature $T$ and the horizontal wind field $\vec{V} \equiv(U, V)$ are two important atmospheric variables and they allow to define Available Enthalpy (EA) and Kinetic Energy (KE) associated with $I V$ (Nikiéma and Laprise 2013) and $T V$ (Clément et al. 2016), calculated as:

$A_{I V}=\frac{c_{p}}{2 T_{r}} \overline{\left\langle T^{2}\right\rangle} \equiv \frac{c_{p}}{2 T_{r}} \overline{\sigma_{T}^{2}}$ and $K_{I V}=\frac{1}{2} \overline{\left\langle\overrightarrow{V^{\prime}} \cdot \vec{V}^{\prime}\right\rangle} \equiv \frac{1}{2}\left(\overline{\sigma_{U}^{2}}+\overline{\sigma_{V}^{2}}\right)$ 


$$
A_{T V}=\frac{c_{p}}{2 T_{r}} \overline{T^{\prime \prime 2}} \equiv \frac{c_{p}}{2 T_{r}} \sum_{T_{n}}^{2} \text { and } K_{T V}=\frac{1}{2} \overrightarrow{\overrightarrow{V^{\prime \prime}} \cdot \overrightarrow{V^{\prime \prime}}} \equiv \frac{1}{2}\left(\sum_{U_{n}}^{2}+\sum_{V_{n}}^{2}\right)
$$

Here $T_{r}$ is a constant reference temperature, which is chosen so that its inverse corresponds to the inverse of $T$ averaged over the time and domain of interest (Marquet 1991). In this study, the reference temperatures will be set to $260^{\circ} \mathrm{K}$ as in Nikiéma and Laprise (2015) for the same regional domain of interest (eastern North America, see Fig. 1c). It is noteworthy that the formulation of $T V$ energies in Eq. (4) is defined for each simulation $n$; hence the ensemble-mean of these energies will be considered in order to compare with $I V$ energies.

(a) Era-Interim: $\mathbf{A}_{\mathrm{TV}}$ in DJF

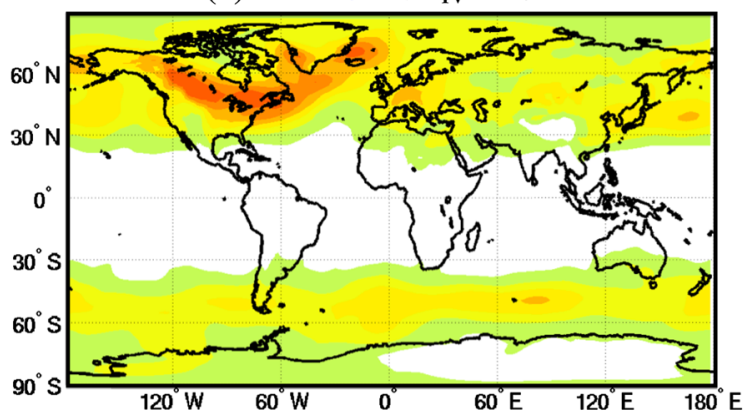

(b) GCM: Ensemble-Mean $\mathbf{A}_{\mathrm{TV}}$ in DJF

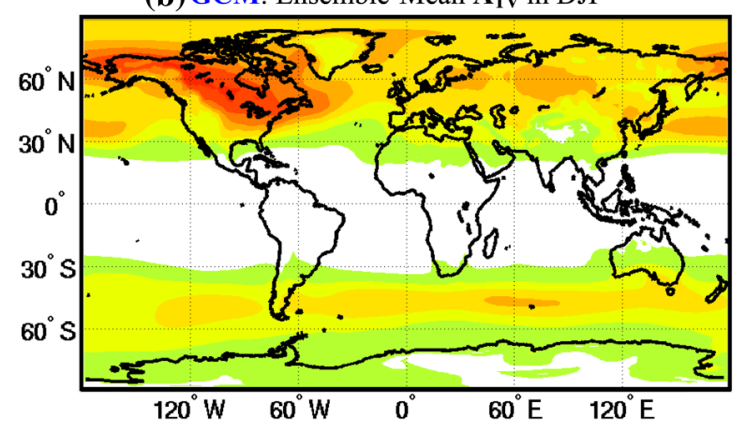

(c) GCM: $A_{I V}$ in DJF

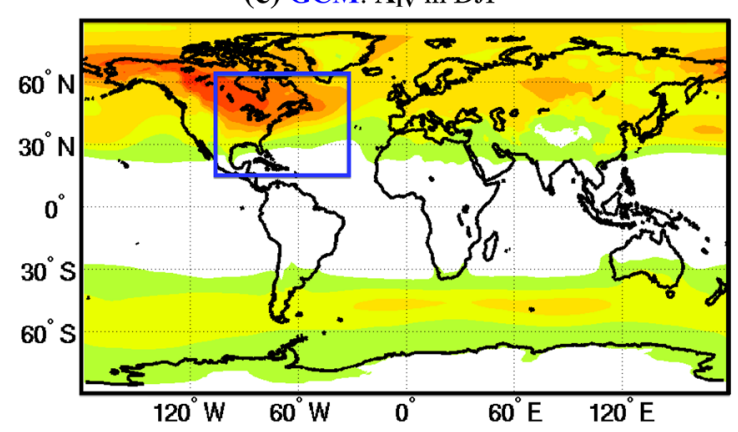

Fig. 1 Maps of vertically integrated available enthalpy associated with transient-eddy variability $\left(\mathrm{A}_{\mathrm{TV}}\right)$ in reanalysis (top row) and GCM simulations (second row), and associated with inter-member

\subsection{Ergodicity in climate simulations}

One goal of this study is to analyse and compare the statistics of TV and IV energies using GCM and RCM simulations. For dynamical systems such as global models of the atmosphere, the ergodic property implies that TV and IV should converge to the same value for a large-member ensemble and over a long time period, i.e.

$\overline{\left\langle T^{\prime 2}\right\rangle} \approx \overline{T^{\prime \prime 2}} \Rightarrow A_{I V} \approx A_{T V}$
$\overline{\left\langle\overrightarrow{V^{\prime}} \cdot \overrightarrow{V^{\prime}}\right\rangle} \approx \overline{\overrightarrow{V^{\prime \prime}} \cdot \overrightarrow{V^{\prime \prime}}} \Rightarrow K_{I V} \approx K_{T V}$

In the results Sect. 3, TV from Era-interim (Dee et al. 2011) reanalyses will be compared to TV and IV calculated (d) Era-Interim: $\mathbf{A}_{\mathrm{TV}}$ in JJA

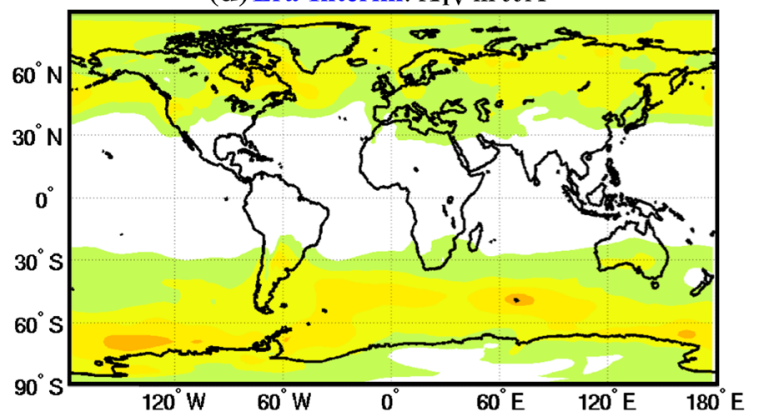

(e) GCM: Ensemble-Mean $\mathbf{A}_{\mathrm{TV}}$ in JJA

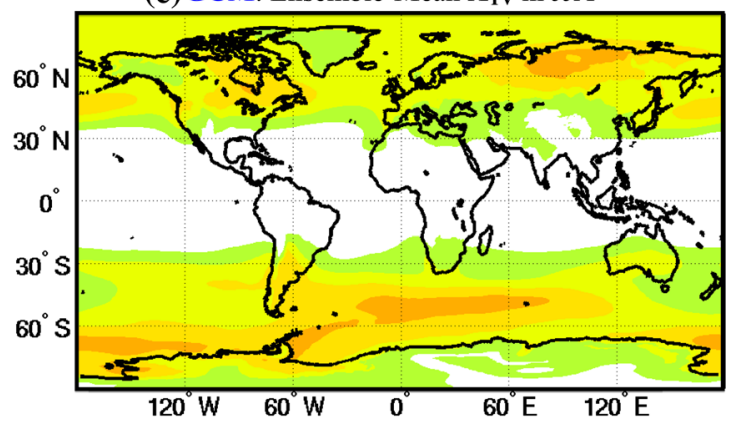

(f) GCM: $A_{I V}$ in JJA

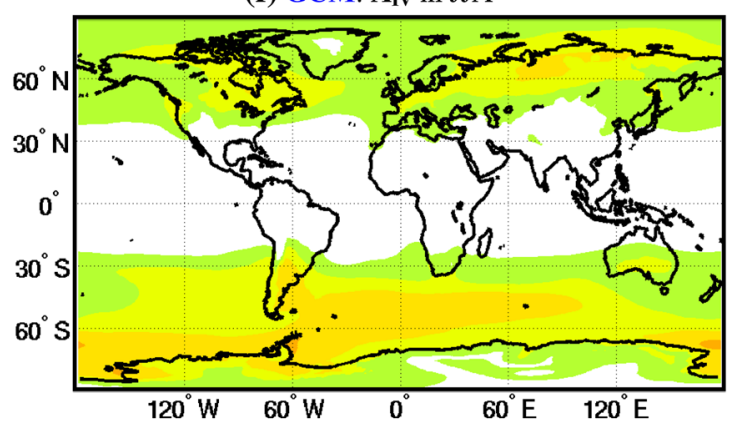

variability $\left(\mathrm{A}_{\mathrm{IV}}\right)$ in $\mathrm{GCM}$ simulations (third row), in DJF (left column) and JJA (right column). The blue rectangle in panel c represents the regional domain of interest. Units: $10^{5} \mathrm{~J} \mathrm{~m}^{2}$

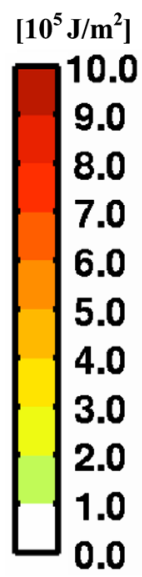


on simulations ensembles from a reanalyses-driven RCM and from a GCM.

\subsection{Budget equations of energies associated with TV and IV}

The energy budget equations used in this work were developed in Nikiéma and Laprise (2013) for the IV energy cycle in ensemble of RCM simulations, and in Clément et al. (2016) for the TV energy in a 1-month RCM simulation. As this study focuses on the analysis of the TV and IV variabilities, the reservoirs associated with background state, namely time- and ensemble-mean, will not be considered.

Following the above two references, the variability energy prognostic equations $\left(L_{E}=\partial E / \partial t\right.$, with $\left.E \in\left\{A_{I V}, A_{T V}, A_{I V}, A_{T V}\right\}\right)$ are written as follows:

$$
\begin{aligned}
& L_{A_{I V}}=R_{A_{I V}}=C_{A_{I V}}-C_{I V}+G_{I V}-F_{A_{I V}}-H_{A_{I V}} \\
& L_{K_{I V}}=R_{K_{I V}}=C_{K_{I V}}+C_{I V}-D_{I V}-F_{K_{I V}}-H_{K_{I V}} \\
& L_{A_{T V}}=R_{A_{T V}}=C_{A_{T V}}-C_{T V}+G_{T V}-F_{A_{T V}}-H_{A_{T V}} \\
& L_{K_{T V}}=R_{K_{T V}}=C_{K_{T V}}+C_{T V}-D_{T V}-F_{K_{T V}}-H_{K_{T V}}
\end{aligned}
$$

destruction of $\mathrm{KE}\left(D_{T V}\right.$ and $\left.D_{I V}\right)$ by dissipation processes. The terms $C_{A}\left(C_{A_{T V}}\right.$ and $\left.C_{A_{I V}}\right)$ and $C_{K}\left(C_{K_{T V}}\right.$ and $\left.C_{K_{I V}}\right)$ are conversion terms from the background (time- and ensemblemean) state to perturbation states for $A E$ and $K E$, respectively. The terms $C_{T V}$ and $C_{I V}$ express the baroclinic energy conversions from $A E$ to $K E$ due to perturbation IV and TV, respectively. The other terms $\left(F_{E}\right.$ and $\left.H_{E}\right)$ are boundary flux terms of energy $E$, exchanged between the regional domain of interest and the external environment; on a global domain, these terms should vanish, unlike on a regional domain.

\subsection{Experiment design and simulations}

This study is done using two ensembles of $N$-member of simulations from a GCM and a RCM, and one dataset from Era-Interim reanalyses (Dee et al. 2011) that is considered as reference and used to drive the RCM. The models used are the fifth-generation Canadian RCM (CRCM5) and its global version referred to as GEM-Global. CRCM5 is based on a limited-area configuration of the GEM3 model (Bélair et al. 2005, 2009) used for numerical weather prediction by the Canadian Meteorological Centre (CMC); the land-surface scheme however is the version 3.5 of the Canadian LAnd Surface Scheme (CLASS; Verseghy

where

$$
\begin{aligned}
& G_{I V}=\overline{l\left\langle T^{\prime} Q^{\prime}\right\rangle} / T_{r} \quad \text { where } l=T_{r} /\langle T\rangle \\
& D_{I V}=-\overline{\left\langle\overrightarrow{V^{\prime}} \cdot \overrightarrow{F^{\prime}}\right\rangle} \text {; } \\
& G_{T V}=\overline{l T^{\prime \prime} Q^{\prime \prime}} / T_{r} \quad \text { where } l=T_{r} / \bar{T} \text {; } \\
& D_{T V}=-\overline{\overline{V^{\prime \prime}} \cdot \overline{F^{\prime \prime}}} \\
& C_{I V}=-\overline{\left\langle\omega^{\prime} \alpha^{\prime}\right\rangle} ; \\
& C_{A_{I V}}=-\frac{C_{p}}{T_{r}}\left[\overline{\left\langle\overrightarrow{V^{\prime}} T^{\prime}\right\rangle} \cdot \vec{\nabla}\langle T\rangle+\overline{\left\langle\omega^{\prime} T^{\prime}\right\rangle} \frac{\partial\langle T\rangle}{\partial p}\right] ; \\
& C_{T V}=-\overline{\omega^{\prime \prime} \alpha^{\prime \prime}} \text {; } \\
& C_{K_{I V}}=-\overline{\left\langle\overrightarrow{V^{\prime}} \cdot\left(\overrightarrow{V^{\prime}} \cdot \vec{\nabla}\right)\langle\vec{V}\rangle\right\rangle}-\overline{\left\langle\overrightarrow{V^{\prime}} \cdot\left(\omega^{\prime} \frac{\partial\langle\vec{V}\rangle}{\partial p}\right)\right\rangle} ; C_{K_{T V}}=-\overrightarrow{\overrightarrow{V^{\prime \prime}} \cdot\left(\overrightarrow{V^{\prime \prime}} \cdot \vec{\nabla}\right) \overline{\bar{V}}}-\overrightarrow{\overrightarrow{V^{\prime}} \cdot\left(\omega^{\prime} \frac{\overline{\vec{V}}}{\partial p}\right)} \\
& F_{A_{I V}}=\vec{\nabla} \cdot\left(\overline{\langle\vec{V}\rangle} A_{I V}\right)+\frac{\partial\left(\overline{\langle\omega\rangle} A_{I V}\right)}{\partial p} ; \\
& F_{A_{T V}}=\vec{\nabla} \cdot\left(\overline{\vec{V}} A_{T V}\right)+\frac{\partial\left(\bar{\omega} A_{T V}\right)}{\partial p} \\
& F_{K_{I V}}=\vec{\nabla} \cdot\left(\overline{\langle\vec{V}\rangle} K_{I V}\right)+\frac{\partial\left(\overline{\langle\omega\rangle} K_{I V}\right)}{\partial p} ; \\
& F_{K_{T V}}=\vec{\nabla} \cdot\left(\overline{\vec{V}} K_{T V}\right)+\frac{\partial\left(\bar{\omega} K_{T V}\right)}{\partial p}
\end{aligned}
$$

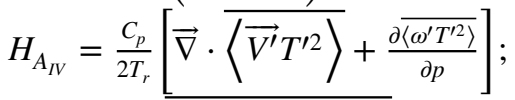

$$
\begin{aligned}
& H_{A_{T V}}=\frac{C_{p}}{2 T_{r}}\left[\vec{\nabla} \cdot\left(\overline{\overline{V^{\prime \prime}} T^{\prime \prime 2}}\right)+\frac{\partial \overline{\omega^{\prime \prime} T^{\prime \prime 2}}}{\partial p}\right] \\
& H_{K_{I V}}=\vec{\nabla} \cdot \overline{\left\langle\left(k_{I V}+\Phi^{\prime}\right) \overrightarrow{V^{\prime}}\right\rangle}+\frac{\partial \overline{\left\langle\left(k+\Phi^{\prime}\right) \omega^{\prime}\right\rangle}}{\partial p} ; \\
& H_{K_{T V}}=\vec{\nabla} \cdot \overline{\left(k_{T V}+\Phi^{\prime \prime}\right) \overline{V^{\prime \prime}}}+\frac{\partial \overline{\left(k+\Phi^{\prime \prime}\right) \omega^{\prime \prime}}}{\partial p}
\end{aligned}
$$

where $l$ is a factor of order unity factor that depends on the choice of the $T_{r}$ value, and $k_{I V}=\left(U^{\prime 2}+V^{\prime 2}\right) / 2$ and $k_{T V}=\left(U^{\prime \prime 2}+V^{\prime \prime 2}\right) / 2$ are kinetic energies associated with variabilities.

Schematically, the above equations constitute two different energy cycles associated with the TV and IV variabilities, as shown in Fig. 12. The terms $G$ represent diabatic generation of $\mathrm{AE}\left(G_{T V}\right.$ and $\left.G_{I V}\right)$ and he terms D relate to the
2000, 2008). Detailed description of CRCM5 is given in Hernández-Díaz et al. (2013). The RCM and GCM share the same subgrid-scale physical parameterisations, except for slight differences in convection-related formulation to account for differences in resolution. The study of Girard et al. (2014) established that the absence of vertical staggering of variables in the discretization of GEM3 induces a computational mode that generates noise near the upper lid 
of the model, both in global and regional configurations; enhanced vertical diffusion of the momentum field has often been applied to damp the computational mode near the top of the model. McTaggart-Cowan et al. (2011) also noted the occasional presence of artificial filamentation of the temperature field around the polar vortex near the top of the limited-area version of GEM3; the initial solution to this issue has been to extend the nesting to drive the upper-level temperature field. Recently an alternative fix has been implemented that reduces the problems encountered in both the regional and global versions, consisting in the application of an enhanced horizontal diffusion in the upper levels (currently four levels). Hence there is currently little difference in vertical upper lid of the RCM and GCM used for this study. There is however some enhanced damping applied over the tropical region in the upper part of the GCM domain; but this lies outside the regional focus area of this work.

For all RCM and GCM simulations, sea-surface temperature and sea-ice coverage are prescribed using Era-Interim data after interpolating horizontally to account for the different grid projection and resolution of RCM and GCM. This reanalysis data is also used as the atmospheric LBC for the $\mathrm{RCM}$, with data linearly interpolated in time at each time step of the RCM. Although CRCM5 code offers the option of large-scale spectral nudging, this option was not used in this study in order to allow the IV to fully develop.

The archived data of the 50-member CRCM5 simulations performed by Nikiéma and Laprise (2015) is used. All the RCM simulations were carried out on the same free domain $(260 \times 160$ grid points in the horizontal, excluding the lateral Davies sponge zone and semi-Lagrangian halo), with a grid mesh of $0.3^{\circ}$ on a non-rotated latitude-longitude projection, and 56 terrain-following hybrid levels in the vertical. The top level is at about $10 \mathrm{hPa}$ and the timestep is $12 \mathrm{~min}$. Each member starts at 0000 UTC on different days from 12 October to 30 November 2004, for a total of 50 simulations. All the simulations share exactly the same lateral boundary conditions (LBC) from Era-Interim; this means that variability due to different LBC is excluded in this study. The regional domain of study covers the eastern part of North America domain and the adjacent western North Atlantic Ocean, as indicated by the blue rectangle in Fig. 1c.

A set of 30-member GCM simulations have been performed. The GCM used a regular latitude-longitude grid of $1^{\circ}$ and 64 terrain-following hybrid levels in the vertical, with the top level at $1 \mathrm{hPa}$, and a timestep of $45 \mathrm{~min}$. The GCM integrations started at 0000 UTC on different days from 12 October to 10 November 2004. Only 30 global simulations were performed due to the limited computational resources; other results show however that 30 members are sufficient to capture most of the IV energy.
For both the RCM and the GCM, the only difference between members is the initial condition used to launch the simulations. The simulations are run for 1 year and the data archived at three hourly intervals, from 1 December 2004 at 0300 UTC to 1 December 2005 at 0000 UTC; but we will focus our analysis on two seasons (DJF and JJA). For the study, the simulated fields are interpolated on 19 pressure levels $(1000,975,950,925,900,850,800,700,600,500$, 400, 300, 250, 200, 100, 70, 50, 30 and $10 \mathrm{hPa}$ ), but we will focus our analysis in the troposphere, i.e. from surface to $250 \mathrm{hPa}$.

\section{Results and analysis}

\subsection{TV and IV energies at the global scale}

Figure 1 shows global maps of vertically integrated values of AE associated with TV $\left(A_{T V}\right)$, in DJF (left column) and JJA (right column), for Era-Interim (top line) and the GCM (second line); the third line shows the corresponding maps of vertically integrated values of $\mathrm{AE}$ associated with IV $\left(A_{I V}\right.$ ) in the 30-member GCM ensemble. Figure 2 shows the corresponding maps of $K_{T V}$. For the GCM, $A_{T V}$ and $K_{T V}$ are first computed for each member in the ensemble of simulations, and the ensemble-mean is shown in Figs. 1 and 2.

The GCM $A_{T V}$ and $K_{T V}$ are found to be similar to those of the reanalysis, confirming the overall skill of the model at seasonal and global scales. As expected maximum TV is found in middle latitudes, with maximum amplitude in the winter hemisphere where storms are strongest; very weak $\mathrm{TV}$ is found in the tropical belt (roughly between $30^{\circ} \mathrm{S}$ and $30^{\circ} \mathrm{N}$ ). Maximum $A_{T V}$ occurs over North America in DJF, associated with large thermal contrast between the cold continent and the warm ocean, with values reaching about $8 \times 10^{5} \mathrm{~J} / \mathrm{m}^{2}$. On the other hand maximum values of $K_{T V}$ occur over the oceans, in the circumpolar belt around Antarctica and off the East coasts of the North American and Eurasian continents, in association with the strongest storms tracks in the respective winter, with values reaching about $20 \times 10^{5} \mathrm{~J} / \mathrm{m}^{2}$. Figures 1 and 2 show remarkably similar patterns of IV and TV energies in the GCM simulations, for both $\mathrm{AE}$ and $\mathrm{KE}$, confirming that the ergodic property is adequately reproduced with the 30-member GCM ensemble. Despite the overall similarities between the $A_{T V}$ and $K_{T V}$ fields of Era-Interim and GCM simulations, noteworthy differences are noted over North America and parts of the Atlantic Ocean in winter, reflecting the different storm tracks.

Figures 3 and 4 present corresponding pressure-latitude cross-sections of zonally averaged AE and KE associated with TV and IV, in DJF and JJA. The GCM appears to reproduce fairly well the TV fields of the reanalysis, and the GCM 
(a) Era-Interim: $\mathbf{K}_{\mathrm{TV}}$ in DJF

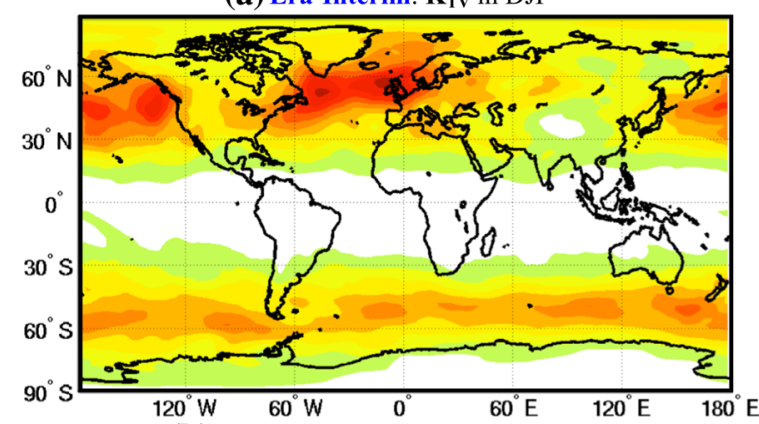

(b) GCM: Ensemble-Mean $\mathbf{K}_{\mathrm{TV}}$ in DJF

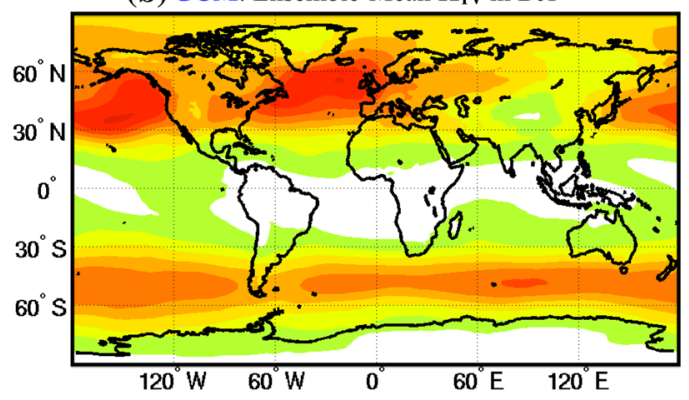

(c) GCM: $\mathrm{K}_{\mathrm{IV}}$ in DJF

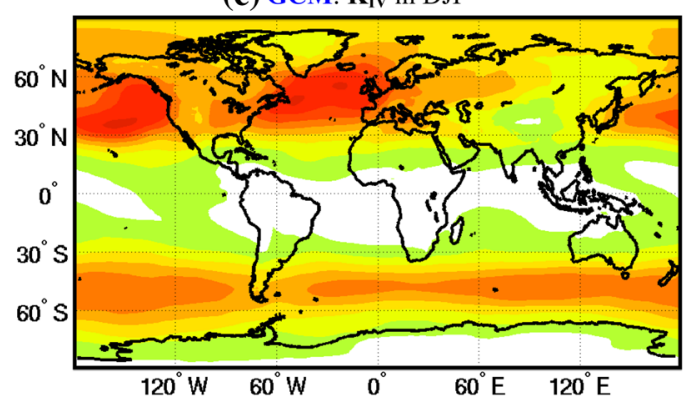

(d) Era-Interim: $\mathbf{K}_{\mathrm{TV}}$ in JJA

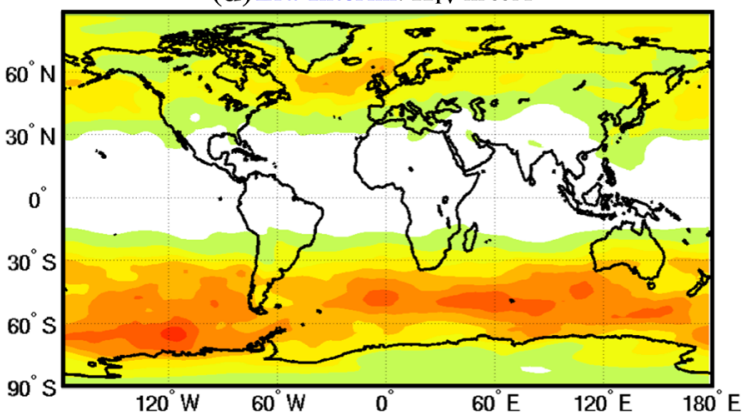

(e) GCM: Ensemble-Mean $\mathbf{K}_{\mathrm{TV}}$ in JJA

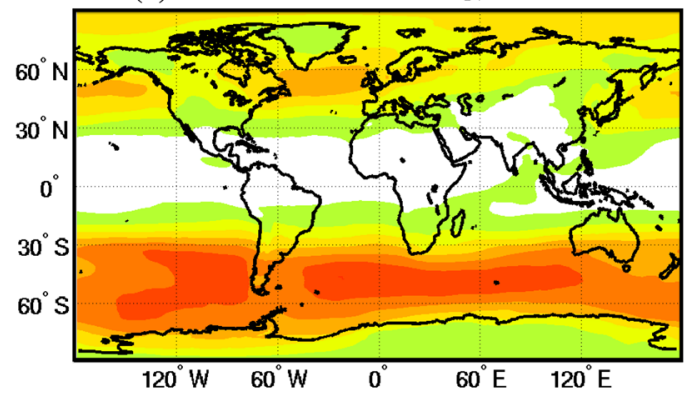

(f) GCM: $\mathrm{K}_{\mathrm{IV}}$ in JJA

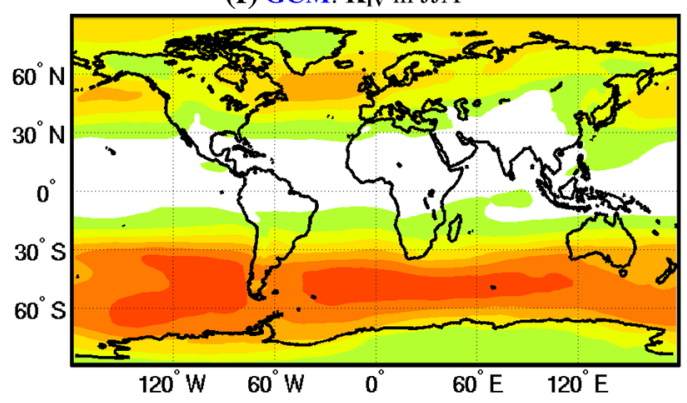

$\left[10^{5} \mathrm{~J} / \mathrm{Kg}\right]$

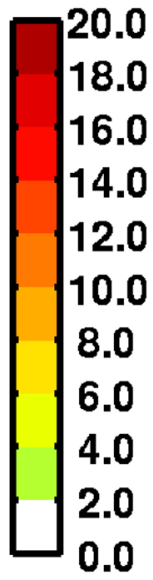

Fig. 2 As in Fig. 1, but for kinetic energy transient-eddy variability $\left(\mathrm{K}_{\mathrm{TV}}\right)$ and inter-member variability $\left(\mathrm{K}_{\mathrm{IV}}\right)$

$\mathrm{TV}$ and IV energies are very similar. Figure 3 shows that $\mathrm{AE}$ energies $\left(A_{T V}\right.$ and $\left.A_{I V}\right)$ are maximum around $60^{\circ}$ of latitude in both hemisphere, with maximum amplitude in winter; in the vertical three maxima appear, one extending from the surface up to about $500 \mathrm{hPa}$, another one at the tropopause near $200 \mathrm{hPa}$, and the third near the top of the displayed domain at $50 \mathrm{hPa}$. Cross-sections in Fig. 4 indicate that the maximum values of $\mathrm{KE}\left(K_{T V}\right.$ and $\left.K_{I V}\right)$ are located at around $250 \mathrm{hPa}$ near $45^{\circ}$ in the two hemispheres, reflecting large variability associated with the jet stream, with largest amplitudes occurring in winter.

\subsection{TV and IV energies at the regional scale}

Figure 5 displays histograms of the $\mathrm{AE}$ and $\mathrm{KE}$ variabilities, averaged over the regional domain of interest indicated by the blue rectangle in Fig. 1c, integrated over the troposphere from the surface to $250 \mathrm{hPa}$, and averaged over the 3-month periods of DJF and JJA. The GCM and
RCM values of $A_{T V}$ and $K_{T V}$ are computed for each member in the ensembles; the red bar shows the 5-95\% percentiles range around the mean, and two black dots represent the minimum and maximum values in the ensemble.

The GCM $A_{T V}$ and $K_{T V}$ values are similar to those of the reanalysis; the RCM values are even closer than those of the GCM owing to the control exerted by the LCB provided by the reanalysis. In DJF, $A_{T V}$ values are about $3.1 \times 10^{5}, 3.5 \times 10^{5}$ and $3.2 \times 10^{5} \mathrm{~J} / \mathrm{m}^{2}$ for reanalysis, $\mathrm{GCM}$ and RCM, respectively, and about $1.4 \times 10^{5}, 1.8 \times 10^{5}$ and $1.6 \times 10^{5} \mathrm{~J} / \mathrm{m}^{2}$ in JJA. The larger winter values reflect the important temperature variability over North America. The $A_{T V}$ values can be translated in equivalent temperature standard deviations of about 4.6, 4.9 and $4.7 \mathrm{~K}$ in winter, and 3.0, 3.4 and $3.2 \mathrm{~K}$ in summer for reanalysis, GCM and RCM, respectively. The corresponding values of $K_{T V}$ are about $8.3 \times 10^{5}, 9.3 \times 10^{5}$ and $8.6 \times 10^{5} \mathrm{~J} / \mathrm{m}^{2}$ in DJF and $3.8,4.8$ and $4.2 \times 10^{5} \mathrm{~J} / \mathrm{m}^{2}$ in JJA. 
(a) Era-Interim:

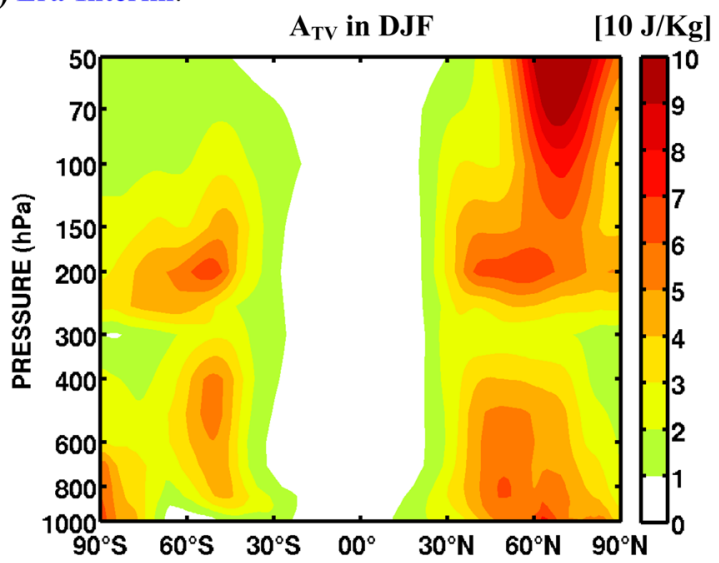

(b) GCM:

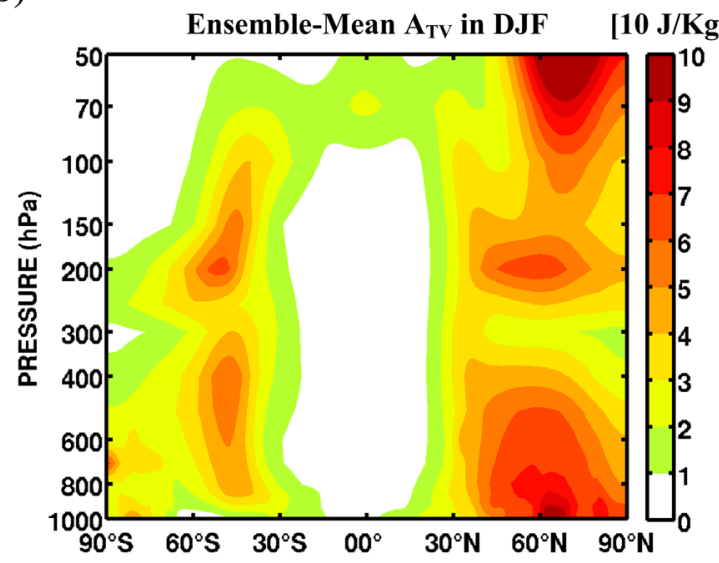

(c) GCM:

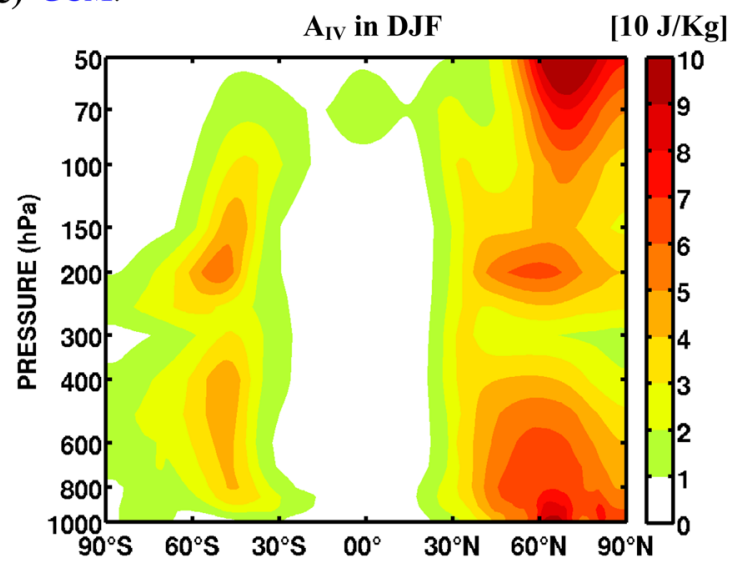

(d) Era-Interim:

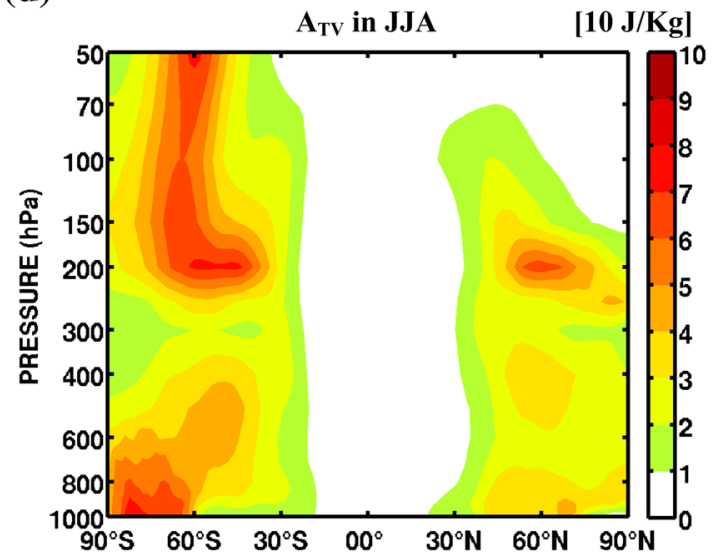

(e) GCM:

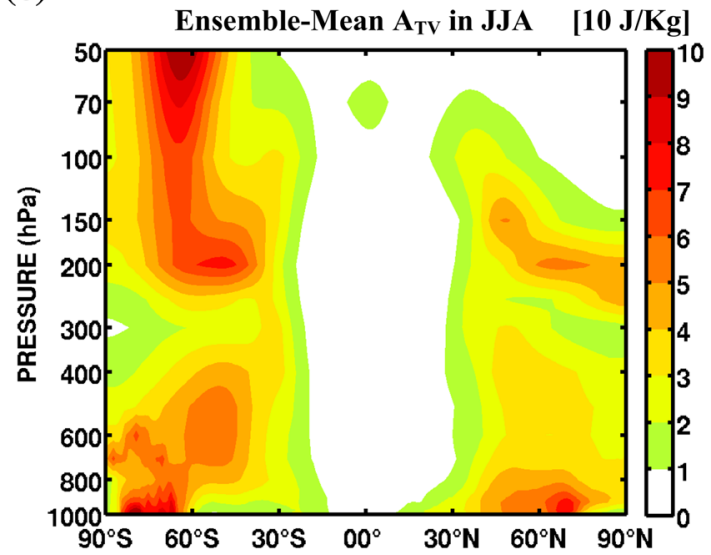

(f) GCM:

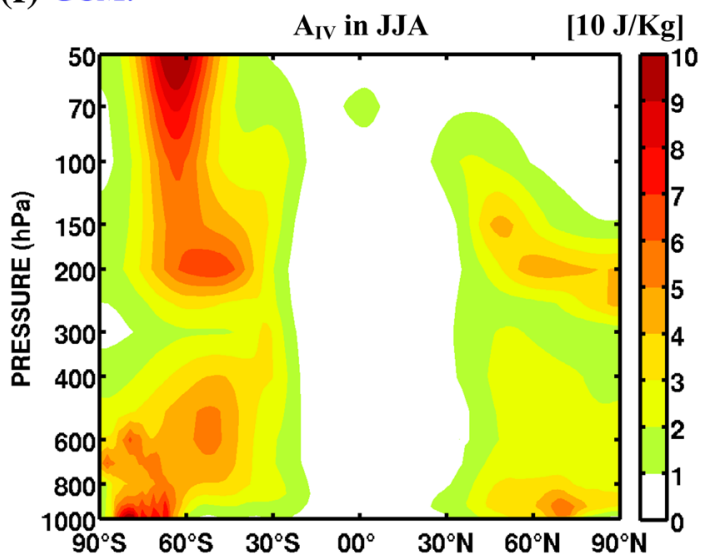

inter-member variability $\left(\mathrm{A}_{\mathrm{IV}}\right)$ in GCM simulations (third row), in DJF (left column) and JJA (right column). Units: $10 \mathrm{~J} \mathrm{~kg}^{-1}$

are identical for all members in the RCM ensemble. For example, while in DJF, GCM's $A_{I V}$ and $K_{I V}$ have intensities of about $3.3 \times 10^{5}$ and $9.4 \times 10^{5} \mathrm{~J} / \mathrm{m}^{2}$, the RCM's values are only $0.2 \times 10^{5}$ and $0.7 \times 10^{5} \mathrm{~J} / \mathrm{m}^{2}$. Similarly in JJA, $A_{I V} \approx 1.3 \times 10^{5} \mathrm{~J} / \mathrm{m}^{2}$ and $K_{I V} \approx 4.7 \times 10^{5} \mathrm{~J} / \mathrm{m}^{2}$ for the $\mathrm{GCM}$ and of $A_{I V} \approx 0.3 \times 10^{5} \mathrm{~J} / \mathrm{m}^{2}$ and $K_{I V} \approx 1.3 \times 10^{5} \mathrm{~J} / \mathrm{m}^{2}$ for the 
(a) Era-Interim:

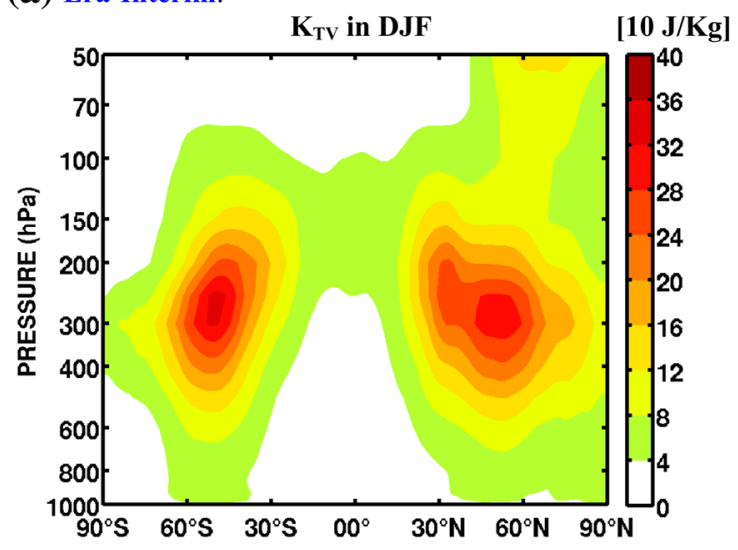

(b) GCM:

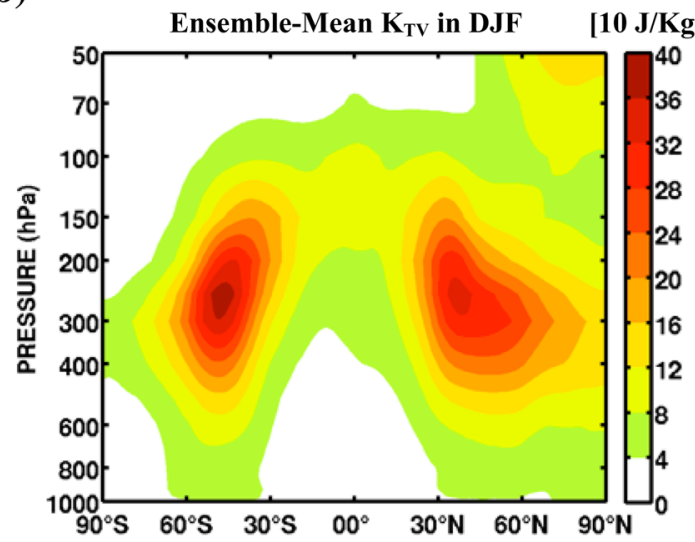

(c) GCM:

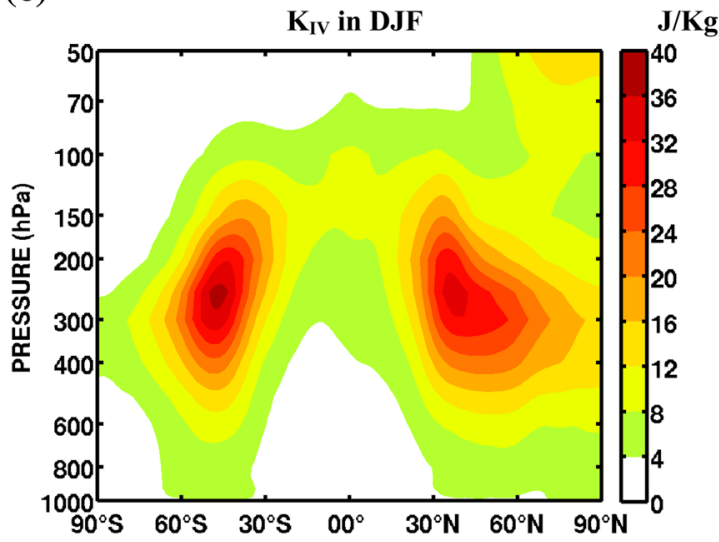

(d) Era_Interim:

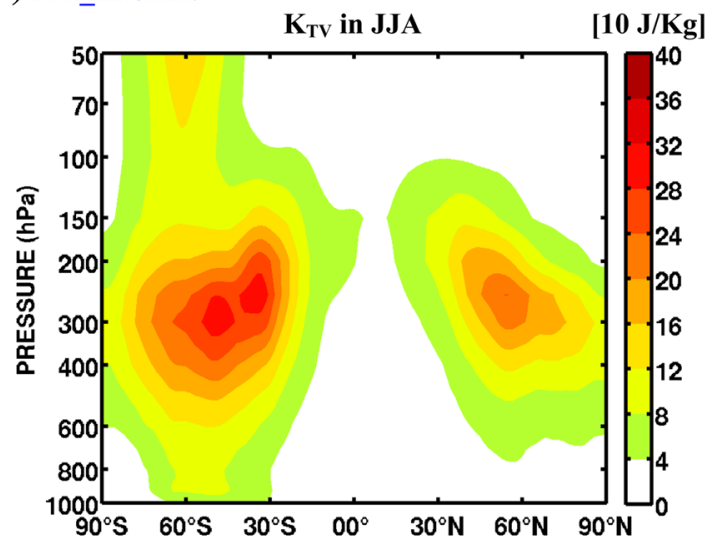

(e) GCM:

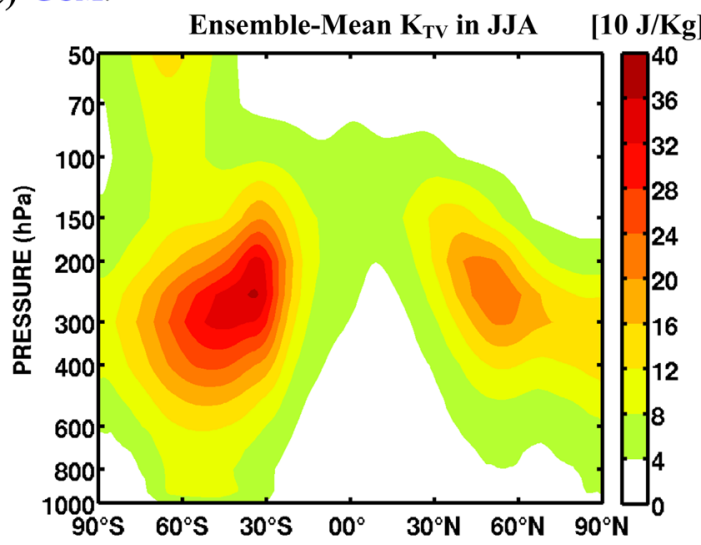

(f) GCM:

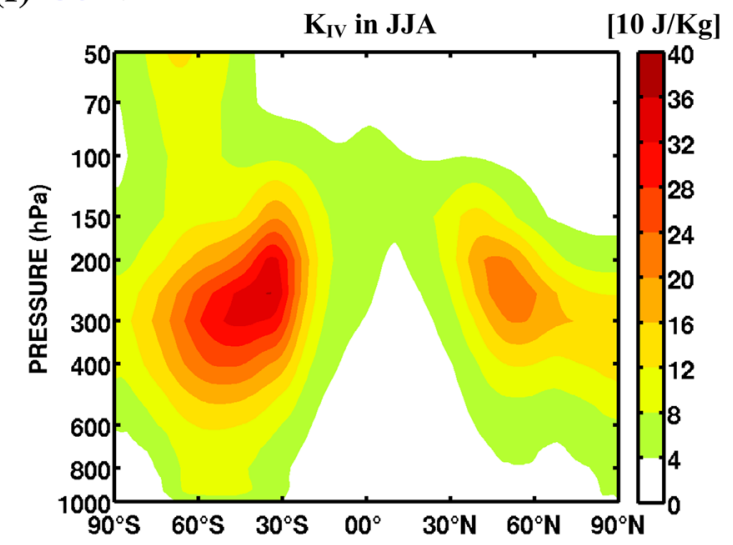

Fig. 4 As in Fig. 3, but for kinetic energy transient-eddy variability $\left(\mathrm{K}_{\mathrm{TV}}\right)$ and inter-member variability $\left(\mathrm{K}_{\mathrm{IV}}\right)$

RCM. As mentioned in previous studies, such as Alexandru et al. (2007), Lucas-Picher et al. (2004, 2008) and Nikiema and Laprise (2011a, b, 2015), these results confirmed that for RCM's IV energies the largest values are found in summer over North America compared to those of winter.

Figure 6 displays the corresponding vertical profiles of variabilities averaged over the regional domain of interest indicated by the blue rectangle in Fig. 1c, for AE (top line) and KE (bottom line), in winter (DJF, left column) and summer (JJA, right column). The profiles of the ensemblemean TV energies for the GCM and RCM are drawn as black and blue continuous lines, respectively, and the gray and blue shaded bands represent the corresponding range of TV energy values. The IV energies for the GCM and RCM are drawn as black and blue dashed lines, respectively. Reanalysis and GCM and RCM simulations exhibit similar vertical profiles of $A_{T V}$, with a first maximum near the surface, around $850-900 \mathrm{hPa}$, and a second maximum near 
(a) DJF: $A_{T V}$ compared to $A_{I V}$

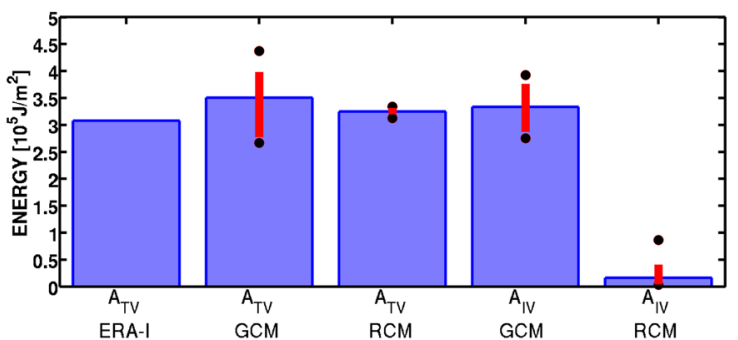

(c)DJF: $K_{\mathrm{TV}}$ compared to $K_{\mathrm{IV}}$

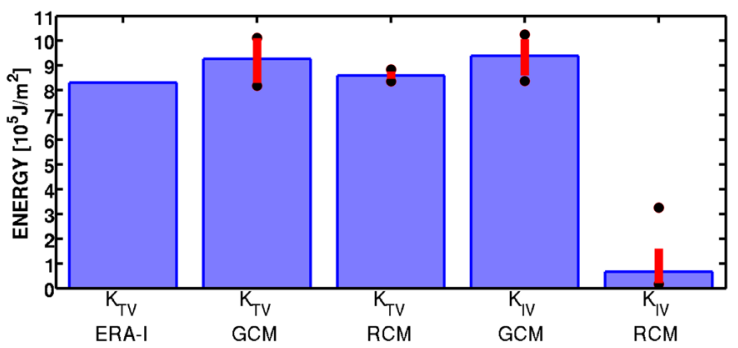

Fig. 5 Average over the domain of interest shown in Fig. 1c of available enthalpy (top row) and kinetic energy (bottom row) associated with transient-eddy variability (subscript TV) and Inter-member Variability (subscript IV), for reanalysis, GCM and RCM simulations, in

150-200 hPa, in both seasons. The profiles of $K_{T V}$ are also fairly similar for the reanalysis and GCM and RCM simulations, exhibiting a single large maximum near the height of the jet stream, around $250 \mathrm{hPa}$. For both TV energies, the RCM profile is closer to the reanalysis one due to the control exerted by the LBC provided by the reanalysis. As far as the IV energies are concerned, the GCM's vertical profiles of IV energies are similar to its TV profiles. While the vertical profiles of GCM-simulated $A_{T V}$ and $K_{T V}$ are similar to those of Era-Interim, we note that the GCM values are generally larger, especially in summer. The RCM IV energies profiles exhibit a similar shape to their TV counterparts, but they are approximately 20 and five times weaker than TV energies in winter and summer, respectively (note the scaling factor applied to RCM IV values).

Figure 7 shows the maps of the vertically integrated (from the surface to $250 \mathrm{hPa}$ ) fields of $A_{T V}, A_{I V}, K_{T V}$ and $K_{I V}$, over the regional domain of interest indicated by the blue rectangle in Fig. 1c, for the reanalysis, GCM and RCM, in winter and summer (note the different scales used for $\mathrm{AE}$ and KE, and for the two seasons). The GCM's TV results are relatively similar to the reanalysis, confirming the skill of the GCM, but the RCM results are even closer due to the control exerted by the LBC. The GCM's IV is quite close to the TV values, confirming the ergodicity property. On the other hand, the RCM's IV is much smaller due to the control exerted by the LBC that imposes vanishing IV at the perimeter of the domain. The last column in Fig. 7 confirms that
(b)JJA: $\mathbf{A}_{\mathrm{TV}}$ compared to $\mathrm{A}_{\mathrm{IV}}$

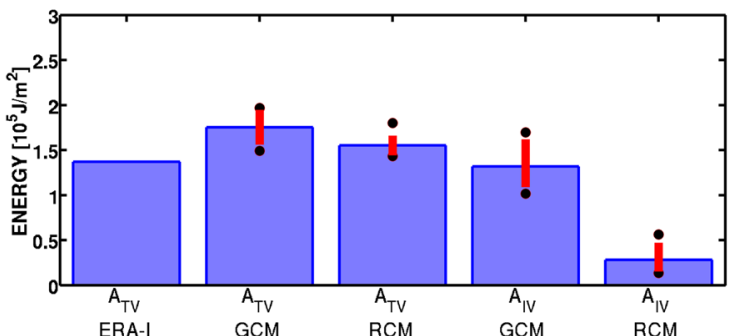

(d) JJA: $K_{\mathrm{TV}}$ compared to $\mathrm{K}_{\mathrm{IV}}$

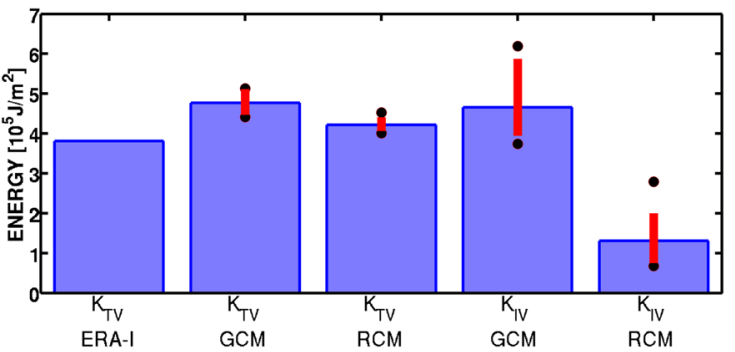

DJF (left column) and JJA (right column). IV energies are computed using ensembles of $30 \mathrm{GCM}$ and $50 \mathrm{RCM}$ simulations; the red bar shows the $5-95 \%$ percentiles range around the mean, and two black dots represent the minimum and maximum values in the ensemble

the RCM's IV energies are transported toward the northeast exit of the regional domain, since the large intensities are found at this location.

Figure 8 shows the time evolution of the GCM and RCM values of $A_{I V}$ and $K_{I V}$, averaged over the regional domain and vertically integrated from the surface to $250 \mathrm{hPa}$. GCM's IV energies exhibit a large seasonal variation, with large values in winter and smaller ones in summer, similarly to the aforementioned seasonal variations of TV energies. The RCM IV energies are much smaller than those of GCM, they reach their maximum values in summer, and they fluctuate rather erratically in time, with sporadic episodes of large growth quickly followed by equally rapid decay.

\subsection{TV and IV energy budgets at the global scale}

Figure 9 displays pressure-latitude cross-sections of the zonally averaged contributions to $\mathrm{A}_{\mathrm{TV}}$ tendency $R_{A_{T V}}$ (Eq. 8) for the reanalysis and GCM, in DJF and JJA. For the GCM, the ensemble-mean fields are used. For the reanalysis, the diabatic generation of transient-eddy Available Enthalpy $\left(G_{T V}\right)$ is evaluated by calculating the residual values using the budget equation of $A_{T V}$ (Eq. 8); this probably explains for a large part the notable differences between the Era-Interim and GCM simulation $G_{T V}$ fields.

In both seasons, and for both reanalysis and GCM, the conversion terms $C_{A_{T V}}$ and $C_{A_{T V}}$ are much larger than all the other contributions to the $A_{T V}$ tendency. The conversion 


\section{$\mathbf{A}_{\mathrm{TV}}$ compared $\mathrm{A}_{\mathrm{IV}}$}

(a) DJF

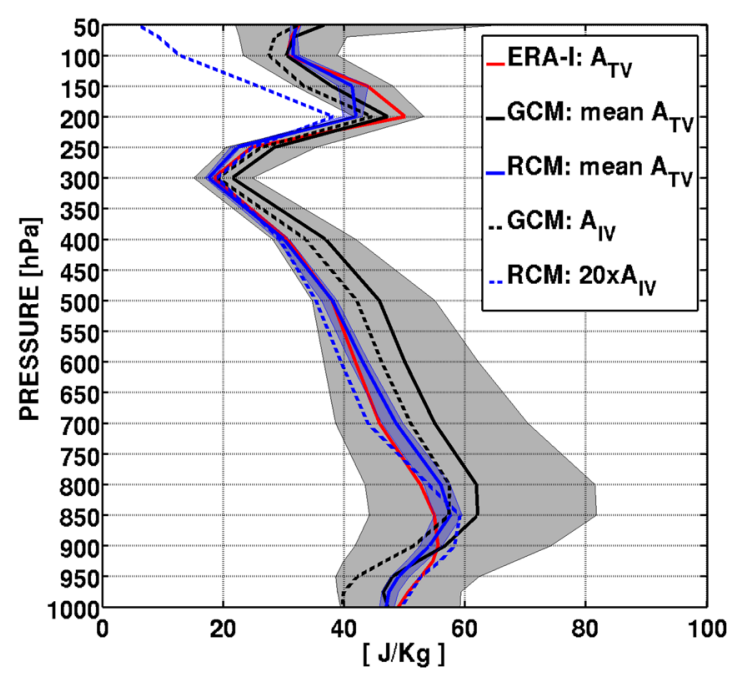

(b) JJA

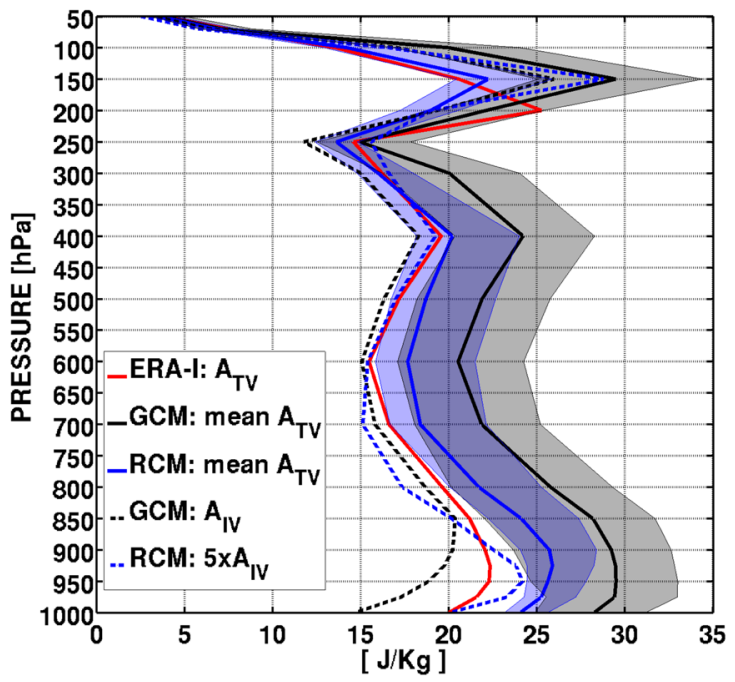

\section{$\mathbf{K}_{\mathrm{TV}}$ compared $\mathbf{K}_{\mathrm{IV}}$}

(c) DJF

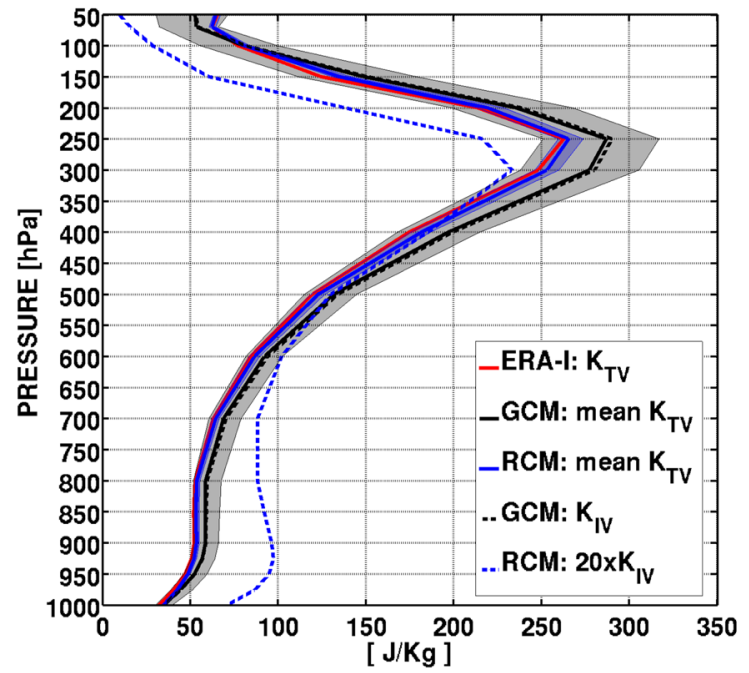

Fig. 6 Vertical profiles of the average over the domain of interest shown in Fig. 1c of available enthalpy (top line) and kinetic energy (bottom line) associated with transient-eddy variability $\left(\mathrm{A}_{\mathrm{TV}}\right.$ and $\mathrm{K}_{\mathrm{TV}}$ ) and inter-member variability $\left(\mathrm{A}_{\mathrm{IV}}\right.$ and $\left.\mathrm{K}_{\mathrm{IV}}\right)$, for reanalysis, GCM and RCM, in DJF (left column) and JJA (right column). The

terms $C_{A_{T V}}$ and $C_{A_{T V}}$ almost mirror one another, with different signs, indicating a high level of compensation in the $A_{T V}$ tendency equation: the $C_{A_{T V}}$ contributes mostly to increasing $A_{T V}$ while $-C_{T V}$ contributes to decreasing it. Both terms exhibit maximum intensities in mid-latitudes troposphere and are largest in the winter hemisphere. The $G_{T V}$ crosssections (first column in Fig. 9) show weak positive contribution to $A_{T V}$ tendency in the middle troposphere, and weak negative contribution near the surface. In the GCM the

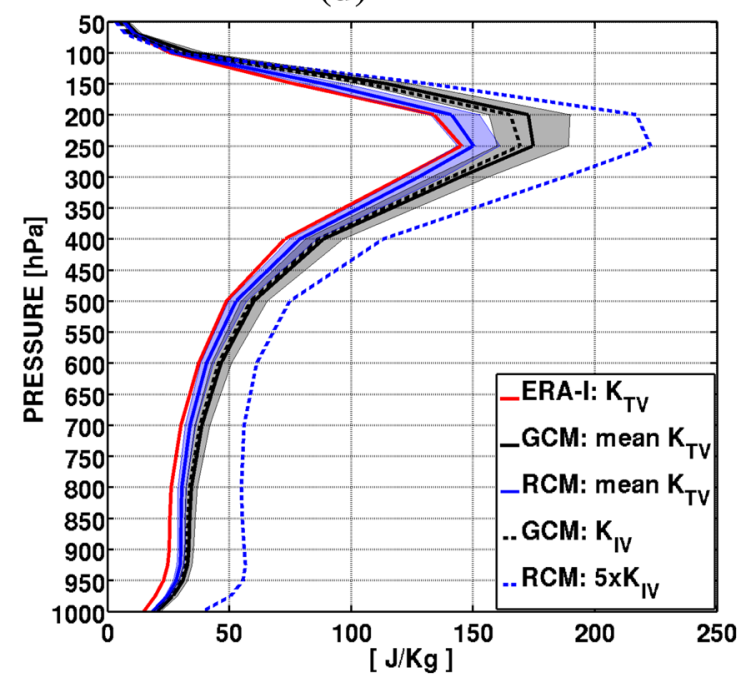

profiles of the ensemble-mean TV energies for the GCM and RCM are drawn as black and blue continuous lines, respectively, and the gray and blue shaded bands represent the corresponding range of TV energy values. The IV energies for the GCM and RCM simulations are drawn as black and blue dashed lines, respectively

positive contributions are due to condensation and convection processes while the negative contributions near the surface are associated with boundary-layer diffusion processes (not shown). The terms $F_{A_{T V}}$ and $H_{A_{T V}}$ should identically vanish when integrated over the entire atmosphere. Indeed, Fig. 9 shows that their zonal averages contribute negligibly to the $A_{T V}$ tendency.

Figure 10 shows the corresponding contributions to $K_{T V}$ tendency $R_{K_{T V}}$ (Eq. 9). For the reanalysis, the term $-D_{T V}$ is 

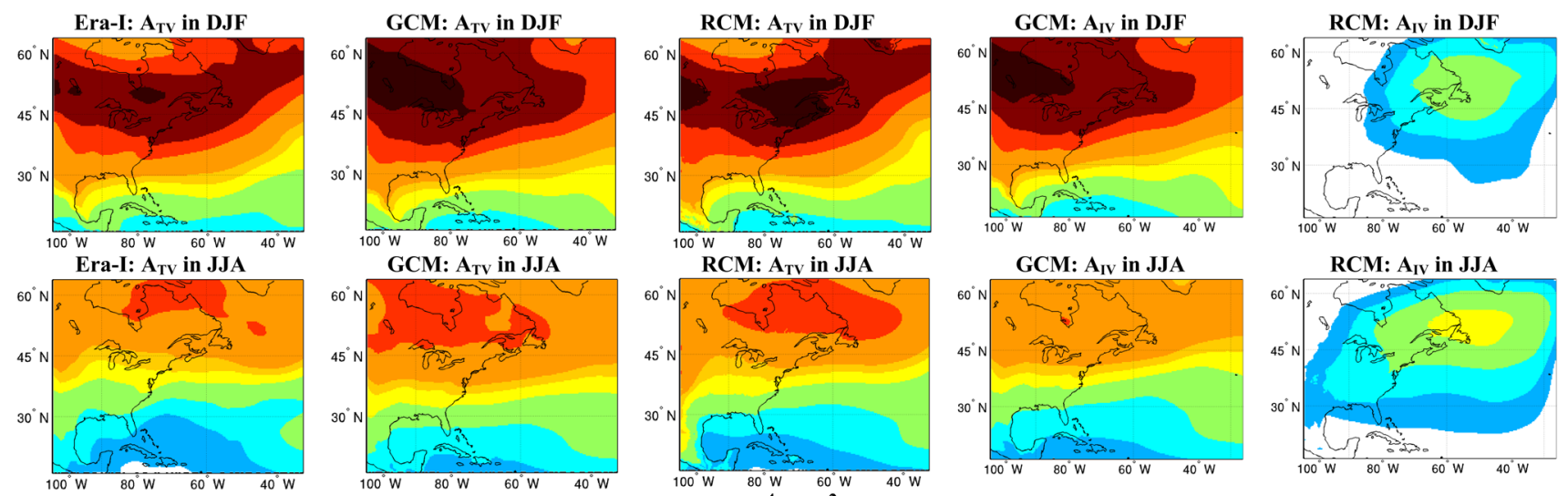

RCM: $\mathbf{A}_{\mathrm{TV}}$ in JJA

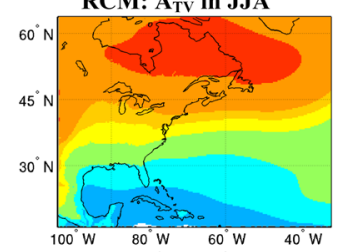

GCM: $A_{\text {IV }}$ in JJA
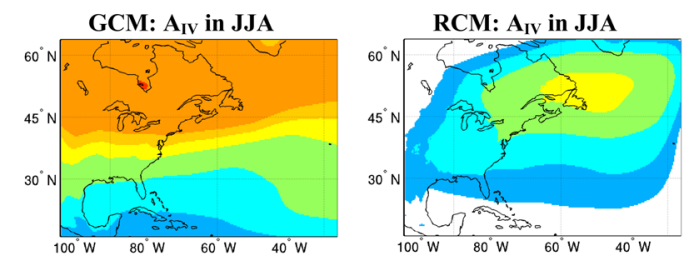

$\left[10^{4} \mathrm{~J} / \mathbf{m}^{2}\right]$
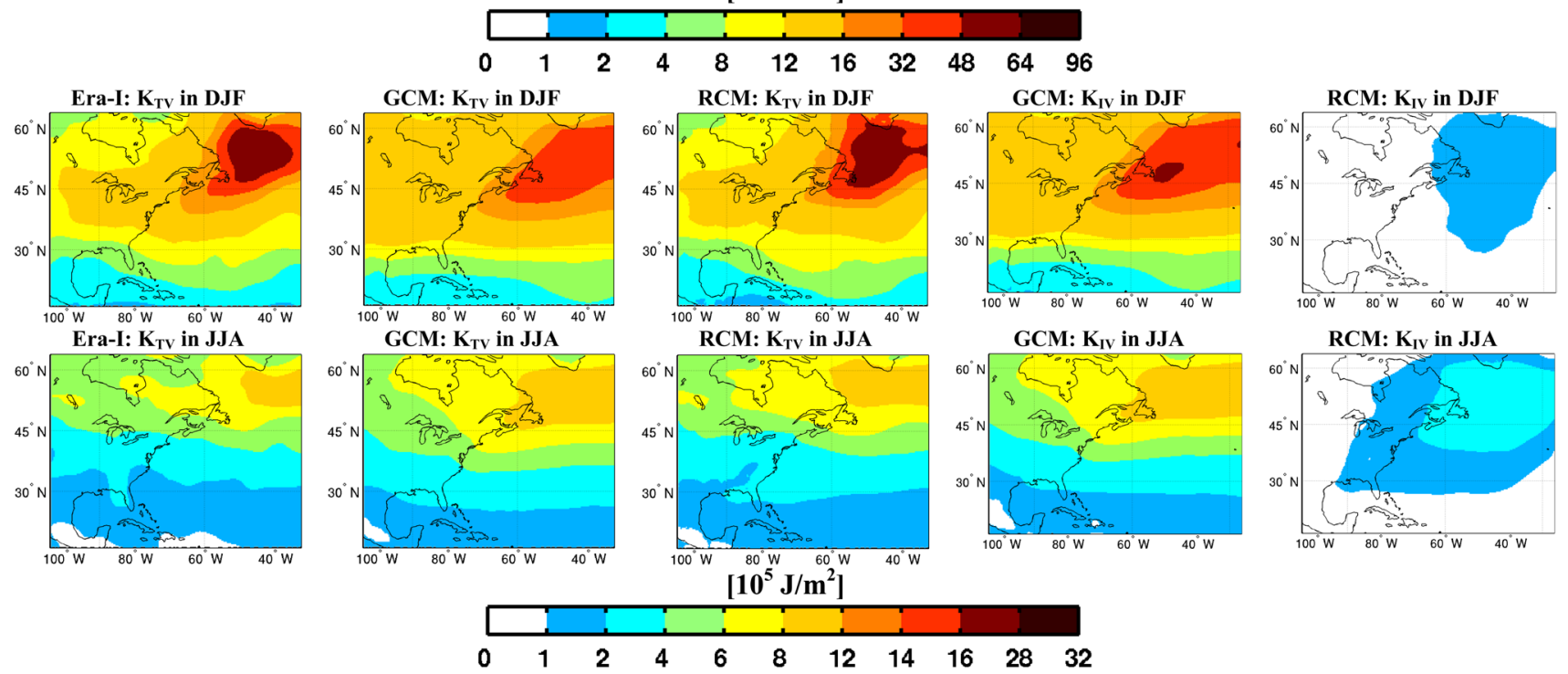

Fig. 7 Maps of vertically integrated available enthalpy and kinetic energy associated with transient-eddy $\left(\mathrm{A}_{\mathrm{TV}}\right.$ and $\mathrm{K}_{\mathrm{TV}}$, respectively) and inter-member variability $\left(\mathrm{A}_{\mathrm{IV}}\right.$ and $\mathrm{K}_{\mathrm{IV}}$, respectively), for reanaly-

evaluated using the estimation of the horizontal momentum sources/sinks $\left(\vec{F}\right.$ in term $\left.D_{T V}\right)$ in the Euler equation. In general the GCM results are very similar to the reanalysis, except for the terms $-D_{T V}$ that show somewhat larger differences due to the fact that they are calculated by residual for the reanalysis. The largest term $C_{T V}$ contributes positively to $K_{T V}$ tendency, with the largest values in the winter hemisphere; in the troposphere, this contribution is mostly offset by a negative contribution from the term $-H_{K_{T V}}$. Near the surface, the positive contributions of $-H_{K_{T V}}$ are offset by the dissipation term $-D_{T V}$. The terms $F_{K_{T V}}$ and $H_{K_{T V}}$ should identically vanish when integrated over the entire atmosphere; Fig. 10 shows that the zonal average of $H_{A_{T V}}$ has substantial values that exhibit large compensation in the vertical.

Figure 11 shows pressure-latitude cross-sections of the zonally averagedss contributions to $A_{I V}$ tendency $R_{A_{V V}}$ (Fig. 11a) and $K_{I V}$ tendency $R_{K_{I V}}$ (Fig. 11b) (Eq. 8) for the sis, GCM and RCM, in DJF (first and third lines) and JJA (second and fourth lines). Units: $10^{4} \mathrm{~J} \mathrm{~m}^{-2}$

GCM, in DJF and JJA. Comparing with corresponding TV fields shown previously in Figs. 9 and 10 confirms very similar results for IV and TV contributions in the 30-member GCM simulations ensemble.

\subsection{TV and IV energy budgets at the regional scale}

In this section, we compare TV and IV energy contributions computed over the regional domain shown as the blue rectangle in Fig. 1c, using data from reanalysis, GCM and RCM. Figure 12 presents each contribution to the AE reservoirs $\left(A_{T V}\right.$ or $\left.A_{I V}\right)$ and $\mathrm{KE}$ reservoirs $\left(K_{T V}\right.$ or $\left.K_{I V}\right)$, in winter (Fig. 12a) and summer (Fig. 12b). For GCM and RCM, the ensemble-mean values of contributions in TV energy budgets are shown; the red bar shows the 5-95\% percentiles range around the mean, and two black dots represent the minimum and maximum values in the ensemble. For a given reservoir, 

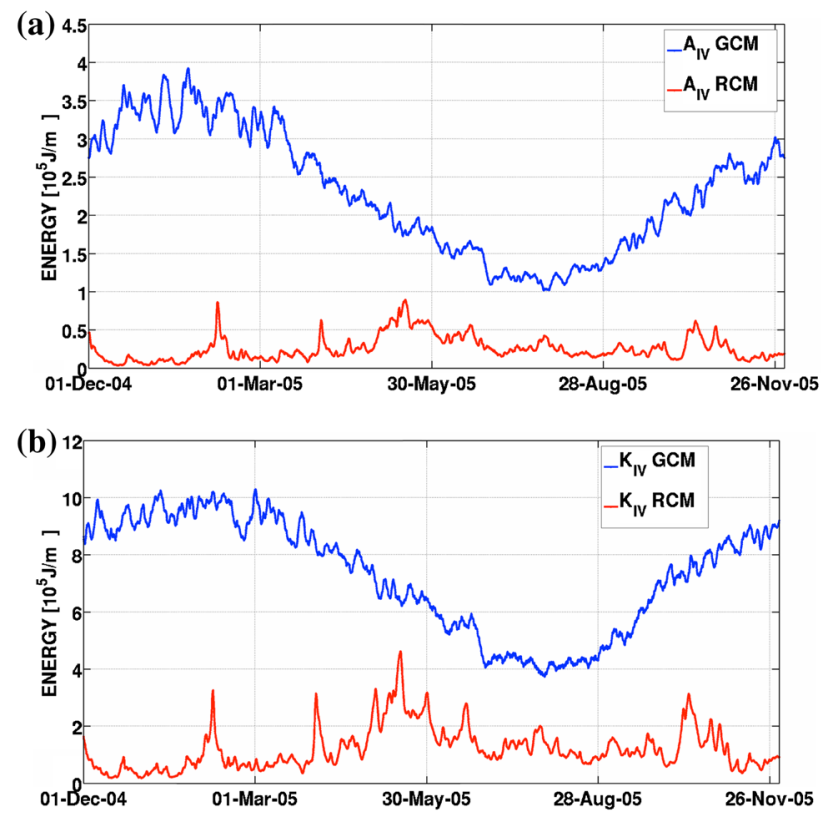

Fig. 8 Time evolution of the average over the regional domain of interest shown in Fig. 1c, of inter-member variability available enthalpy $\left(\mathrm{A}_{\mathrm{IV}}, \mathbf{a}\right)$ and kinetic energy $\left(\mathrm{K}_{\mathrm{IV}}, \mathbf{b}\right)$, in GCM and $\mathrm{RCM}$ ensembles

the ingoing and outgoing arrows indicate the gain and loss of energies, respectively.

Over the regional domain of study, the $A_{T V}$ and $A_{I V}$ reservoirs gain energy mainly by $C_{A_{T V}}$ and $C_{A_{T V}}$ and transfer a similar amount of energy to $K_{T V}$ and $K_{I V}$ via the baroclinic conversion terms $C_{T V}$ and $C_{I V}$, respectively. On the other hand, the $K_{T V}$ and $K_{I V}$ reservoirs loose energy mostly by the dissipation terms $D_{T V}$ and $D_{I V}$, but also somewhat by $H_{K_{T V}}$ and $H_{K_{I V}}$, and a little by $F_{K_{T V}}$ and $F_{K_{I V}}$, respectively. The three datasets exhibit approximately the same intensity for the transient-eddy fluxes $C_{A_{T V}}, C_{T V}$ and $D_{T V}$, with average winter values of about $6.4,6.2$ and $3.4 \mathrm{~W} / \mathrm{m}^{2}$, and average summer values of about $2.5,2.6$ and $1.5 \mathrm{~W} / \mathrm{m}^{2}$, respectively. For the GCM, the terms associated with IV have approximately the same intensity to those of TV. The RCM's IV terms however show much weaker intensities: $C_{A_{I V}}=0.76 \mathrm{~W} /$ $\mathrm{m}^{2}, C_{I V}=0.85 \mathrm{~W} / \mathrm{m}^{2}$ and $D_{I V}=0.46 \mathrm{~W} / \mathrm{m}^{2}$ in winter, and $C_{A_{I V}}=1.0 \mathrm{~W} / \mathrm{m}^{2}, C_{I V}=1.2 \mathrm{~W} / \mathrm{m}^{2}$ and $D_{I V}=0.6 \mathrm{~W} / \mathrm{m}^{2}$ in summer. On average, the conversion term $C_{K_{T V}}$ has a small negative contribution to $K_{T V}$ reservoir for all datasets, as well as $C_{K_{I V}}$ for the GCM, while $C_{K_{I V}}$ for the RCM shows a small positive contribution.

For $A_{T V}$ and $A_{I V}$ reservoirs, the diabatic generation term $\left(G_{T V}\right.$ and $\left.G_{I V}\right)$ and the boundary terms $\left(F_{A_{T V}}, H_{A_{T V}}\right.$ and $F_{A_{I V}}$, $H_{A_{I V}}$ ) have very small magnitude compared to conversion terms in both seasons. We can also note that the generation term $G_{T V}$ for the reanalysis, calculated as a residual, has a different sign than the GCM and RCM in winter and is close to zero in summer. For $K_{T V}$ and $K_{I V}$ reservoirs, the boundary terms $\left(F_{K_{T V}}, H_{K_{T V}}\right.$ and $\left.F_{K_{I V}}, H_{K_{I V}}\right)$ act to reduce energies, and the GCM IV contributions are similar to the $\mathrm{TV}$ values of the three datasets. The terms $F_{K_{I V}}$ and $H_{K_{I V}}$ for the RCM, however, are much smaller in winter, but have similar amplitude in summer. Especially in the case of GCM, the large spreads seem to indicate that some mean values are not significant, notably those of $F_{A_{T V}}$ and $H_{A_{T V}}$ in Fig. 12b and Fig. 12a, respectively.

Figure 13 displays the vertical profiles of horizontal averages of the terms in the $A_{T V}$ budget in winter (first row) and summer (second row). The gray and blue light bands represent the ranges of values for terms associated with TV energy tendencies for the GCM and RCM, and their ensemble-mean profiles are shown in black and blue lines, respectively. The profiles of terms in $A_{T V}$ budget for reanalysis are presented in red lines. The profiles of $A_{I V}$ budget for the GCM and RCM are shown in black and blue dashed lines, respectively.

For terms in $A_{T V}$ budget at the seasonal scale, the results of the GCM and RCM are generally similar to those of the reanalysis. In terms of intensity, results from climate models (GCM and RCM) and Era-Interim are different for $G_{T V}$ in both seasons and for conversion terms $\left(C_{A_{T V}}\right.$ and $\left.C_{T V}\right)$ in summer. As noted earlier, the term $G_{T V}$ is evaluated by calculating the residual values using the budget equation of $A_{T V}$ (Eq. 8) for Era-Interim, which could explain the difference of results when compared with ones of climate models. For $C_{A_{T V}}$ and $C_{T V}$ in summer, results showed that the covariance of temperature and wind perturbations from Era-Interim fields are smaller in the troposphere compared to those of climate models (see Fig. 13: second and third bottom panels) resulting to smaller values for reanalysis conversion terms. This result seems to be associated with small values of reanalysis's $A_{T V}$ and $K_{T V}$ in the troposphere during the summer compared to those of climate models (see Fig. 6b and 6d). The range of values for the RCM terms is smaller than that of the GCM, and it is usually contained within the range of the GCM, except for the term $G_{T V}$ in summer. For the GCM, the terms in $A_{T V}$ and $A_{I V}$ budgets are rather similar, as seen by the proximity of the continuous and dashed black lines. For the RCM however the terms in the $A_{I V}$ budget are much smaller (note the difference of scales), but the vertical profiles are somewhat similar to those of the $A_{T V}$ budget. The conversion terms $C_{A_{T V}}$ and $C_{A_{I V}}$ act as source for $A_{T V}$ and $A_{I V}$ reservoirs, which implies that on average covariance of temperature and wind TV (or IV) fluctuations are downthe-gradient of time- (or -ensemble) mean temperature. The terms $-C_{T V}$ and $-C_{I V}$ act as sink for $A_{T V}$ and $A_{I V}$ reservoirs, which implies that temperature and vertical motion TV (or IV) fluctuations are negatively correlated, meaning that warm perturbations rise and cold perturbation sink (e.g., Nikiéma and Laprise 2011a, b, 2015). 


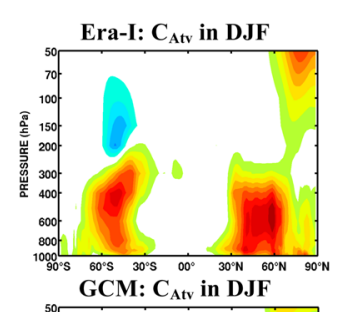

$$
L_{A_{T V}}=R_{A_{T V}}=C_{A_{T V}}-C_{T V}+G_{T V}-F_{A_{T V}}-H_{A_{T V}}
$$
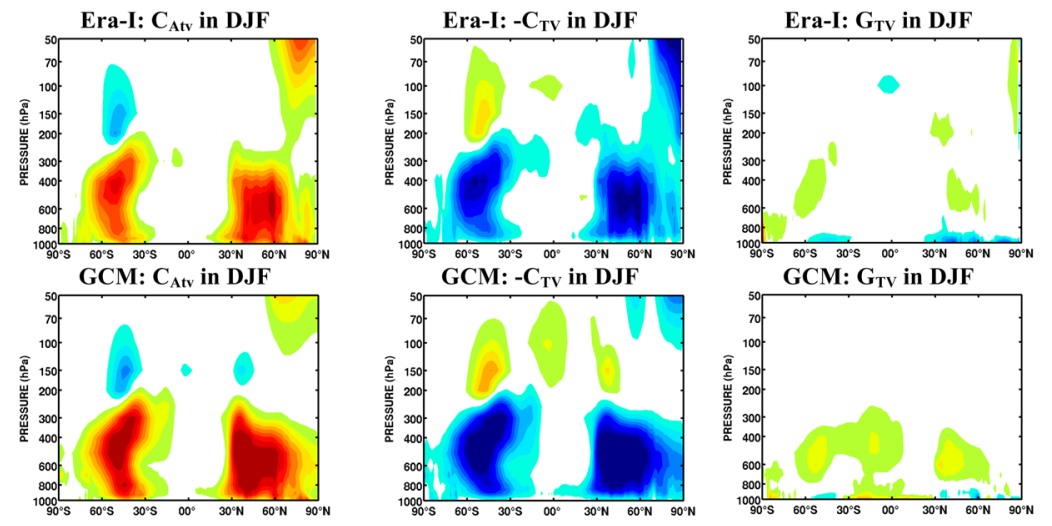

GCM: $G_{T V}$ in DJF
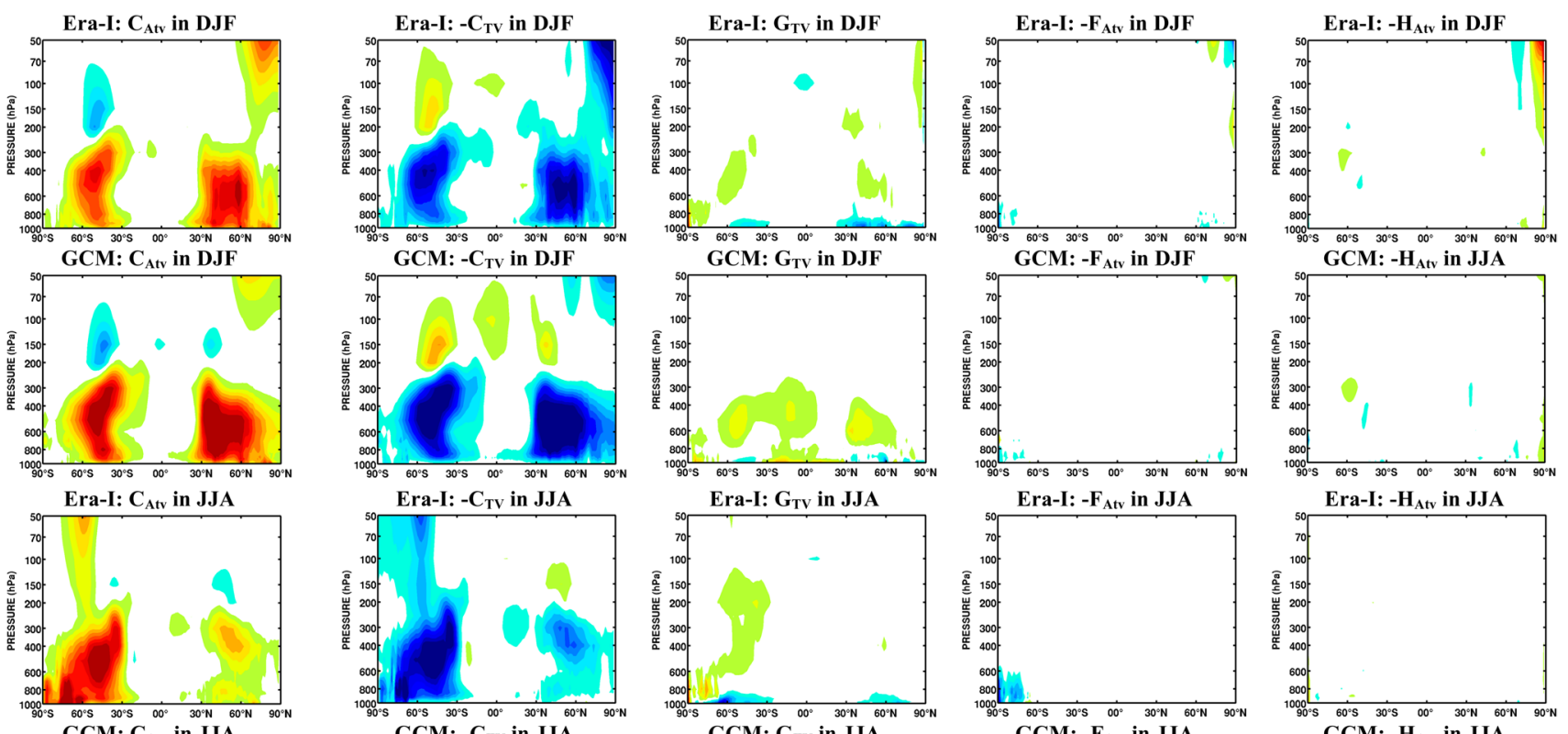

Era-I: $\mathbf{G}_{\mathrm{TV}}$ in JJA
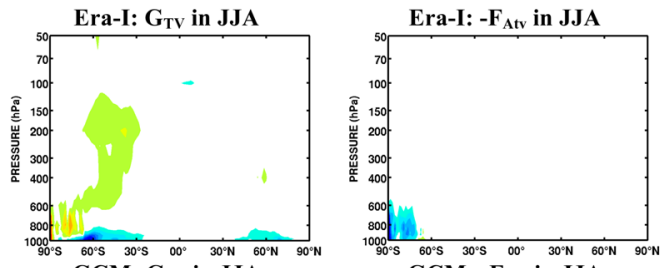
GCM: $-\mathbf{C}_{\mathrm{TV}}$ in JJA
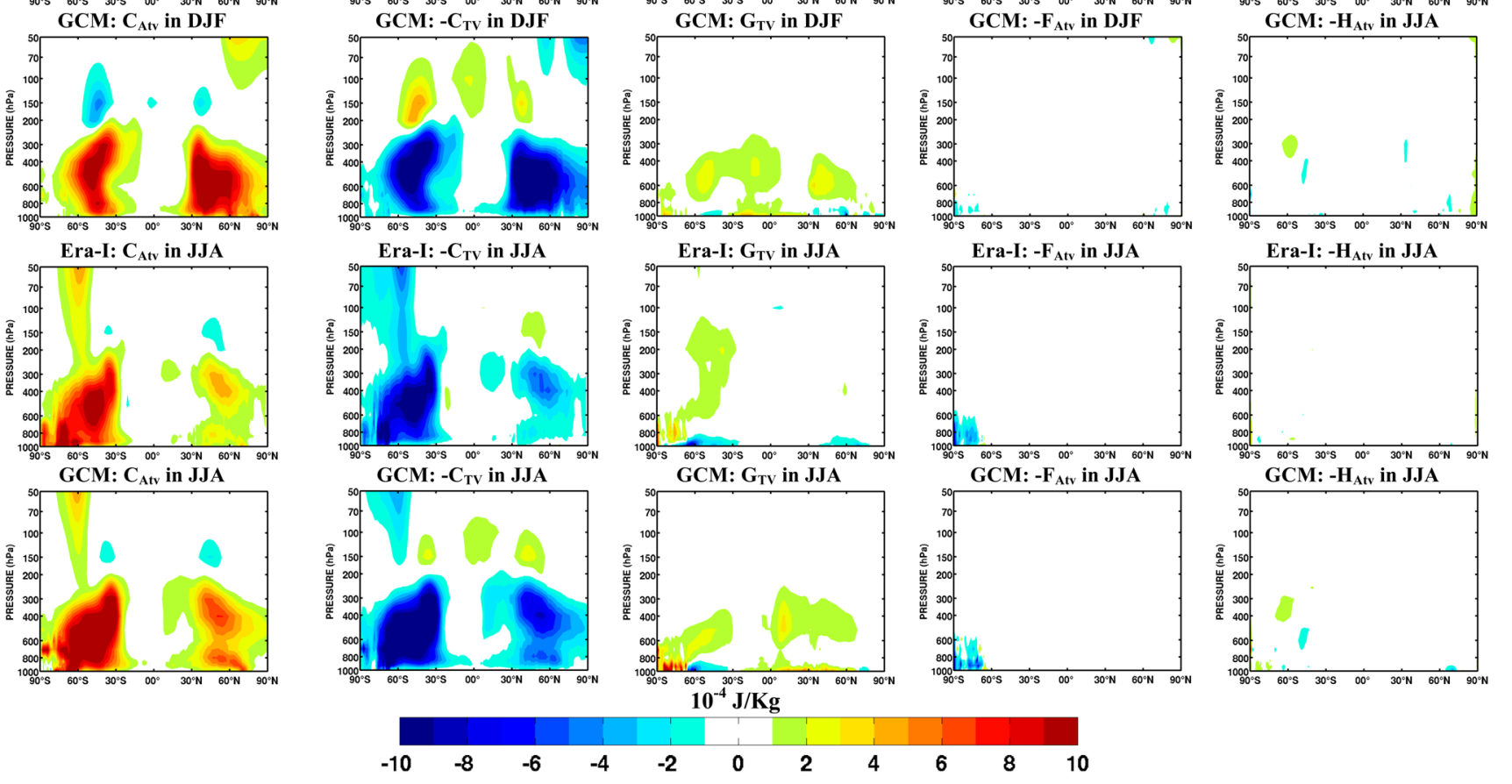
the reanalysis and GCM, in DJF (first two rows-a) and JJA (last two rows-b). Units: $10^{4} \mathrm{~J} \mathrm{Kg}^{-1}$

In winter, the diabatic generation term $G_{T V}$ contributes positively between 250 and $850 \mathrm{hPa}$ and negatively in the lower layers (between $850 \mathrm{hPa}$ and surface) for all the datasets, as well as $G_{I V}$ for the GCM and RCM. The reanalysis profile is similar to that of models, but with weaker positive contribution in the middle troposphere and larger negative contribution near the surface. In summer, the two climate models indicate positive contribution of $G_{T V}$ at all pressure levels, on average, and results from the reanalysis show positive and negative contributions in higher (above $650 \mathrm{hPa}$ ) and lower troposphere, respectively. Physically, the positive sign of terms $G$ means that temperatures TV (or IV) perturbations are positive correlated with diabatic heating perturbations associated with condensation, convection and radiation processes (results not shown); their negative sign near the surface reflect boundary-layer turbulent diffusion processes (results not shown).

The vertical profiles of boundary terms $\left(F\right.$ and $H$ ) of $A_{T V}$ tendency show weak contributions with positive and negative values ranged between $\pm 1 \times 10^{-4} \mathrm{~W} / \mathrm{kg}$, as well as $A_{I V}$ tendency for the GCM. For the RCM, the range of values is about five times smaller. For the RCM, the term $F_{A_{I V}}$ contributes negatively at all pressure levels and in both seasons because $A_{I V}$ energy is transported out of the regional domain (e.g. Nikiéma and Laprise 2011a, b, 2015).

Figure 14 shows the corresponding vertical profiles of horizontally averaged contributions to $K_{T V}$ and $K_{I V}$ budgets, using the same colour convention. In general the TV results of the models are similar to those of the reanalysis, with a larger ranges of values for the GCM than the RCM. The $K_{T V}$ and $K_{I V}$ reservoirs are fed mainly by the baroclinic conversion terms $C_{T V}$ and $C_{I V}$, respectively, which are offset by negative contribution of $-H_{K_{T V}}$ and $-H_{K_{I V}}$ in the middle troposphere, and by the dissipation processes $-D_{T V}$ and $-D_{I V}$ near the surface as a result of Ekman pumping (e.g. Nikiéma and Laprise 2015). The boundary terms $-F_{K_{T V}}$ and $-F_{K_{I V}}$ act mostly negatively, with largest values in the vicinity of the tropopause near the jet stream, as a result of TV or IV kinetic energies being transported outside the regional domain. At the regional scale, the term $C_{K_{T V}}<0$ implies that the $K_{T V}$ energy contributes to reinforce the background kinetic energy mostly near the jet stream. 

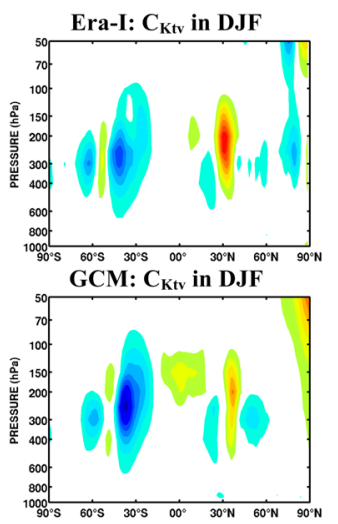

Era-I: $\mathrm{C}_{\mathrm{Ktv}}$ in JJA

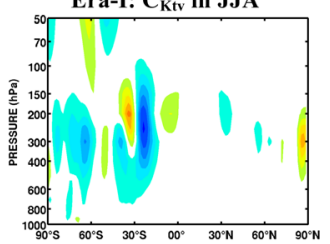

GCM: $\mathrm{C}_{\mathrm{Ktv}}$ in JJA

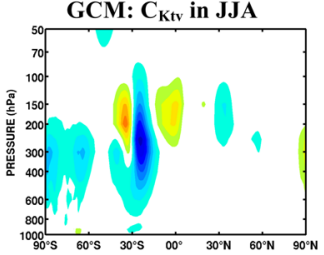

$$
L_{K_{T V}}=R_{K_{T V}}=C_{K_{T V}}-C_{T V}-D_{T V}-F_{K_{T V}}-H_{K_{T V}}
$$
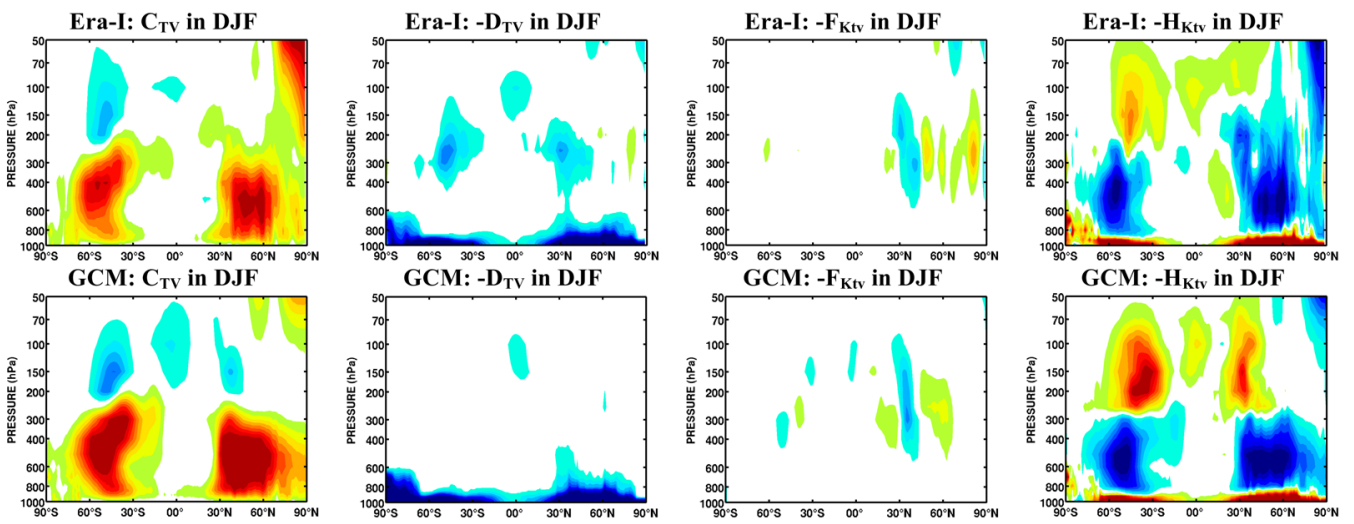

Era-I: - $D_{T V}$ in JJA

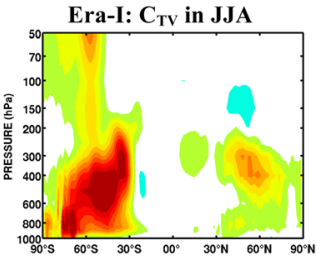

GCM: $\mathrm{C}_{\mathrm{TV}}$ in JJA
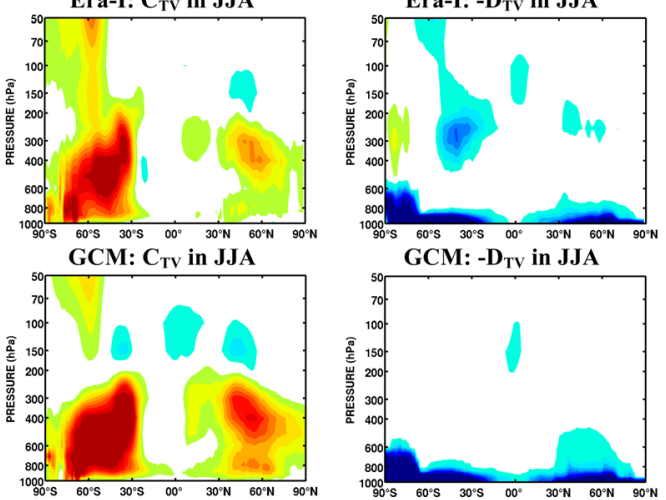

GCM: - $D_{T V}$ in JJA
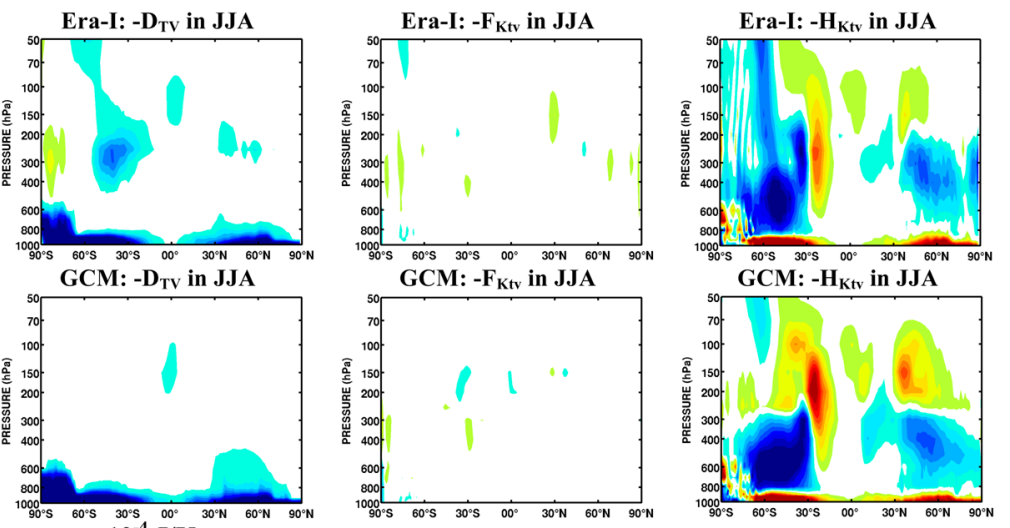

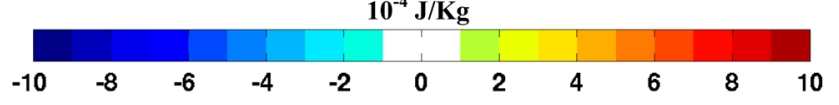

Fig. 10 As in Fig. 9, but for transient-eddy kinetic energy $\left(\mathrm{K}_{\mathrm{TV}}\right)$ tendency $\left(R_{K_{T V}}\right)$ contributions

For the GCM, the IV results are similar to those of the TV. For the RCM, the IV terms have smaller intensity but exhibit generally similar profiles to those of the TV, except for the barotropic conversion term $\left(C_{K_{I V}}>0\right)$ that has positive sign, contrary to GCM's result. This result indicates that this term $\left(C_{K_{I V}}\right)$ acts to feed $K_{I V}$ reservoir from the ensemblemean kinetic energy (Nikiema and Laprise 2015), unlike the case of the GCM where the ensemble-mean kinetic energy is fed by $C_{K_{I V}}$.

In the following paragraphs of this section, the reader is referred to Figures S1 and S2 in the Supplementary Material. Figure S1 displays the maps of contributions, vertically integrated over the whole troposphere (from the surface to $250 \mathrm{hPa}$ ), of all the terms in the $A_{T V}$ budget for the reanalysis, the GCM and RCM, and in the $A_{I V}$ budget for the GCM and RCM, in winter (panel S1a) and summer (panel $\mathrm{S} 1 b$ ); note that different scaling used for some terms. For the climate models, the $A_{T V}$ ensemble-mean contributions are shown.

For the $A_{T V}$ contributions we note again that, while the GCM's contributions are somewhat similar to those of the reanalysis, the RCM's contributions are very close to those of the reanalysis due to the control exerted by the LBC. On the other hand the GCM's $A_{I V}$ contributions are very similar to those of the $A_{T V}$ contributions, reflecting the ergodicity property. Hence the patterns of each IV and TV contributions are similar for all datasets, except for the RCM's $A_{I V}$ contributions.

In all datasets, there are large compensating contributions from terms $C_{A_{T V}}$ and $C_{T V}$ to the $A_{T V}$ budget, with maximum intensity of about $30 \mathrm{~W} / \mathrm{m}^{2}$ along the storm tracks off the east coast in winter, and $10 \mathrm{~W} / \mathrm{m}^{2}$ over Canada in summer. For the GCM, the patterns of these conversions terms are similar to those of $C_{A_{I V}}$ and $C_{I V}$ in the $A_{I V}$ budget. In the $\mathrm{RCM}$, while the $C_{A_{I V}}$ and $C_{I V}$ terms are also largely compensating one another, their patterns are rather different from the corresponding TV terms, reaching their maximum intensity near the northeastern (outflow) part of the domain, with maximum intensities of only about $3 \mathrm{~W} / \mathrm{m}^{2}$.

The diabatic generation term $G_{T V}$ is modest, with maximum values of about $3 \mathrm{~W} / \mathrm{m}^{2}$ in both seasons. In the reanalysis this term is computed as residual, which may explain why its pattern is very noisy and rather different from that of the climate models. There is however a general common 
(a)
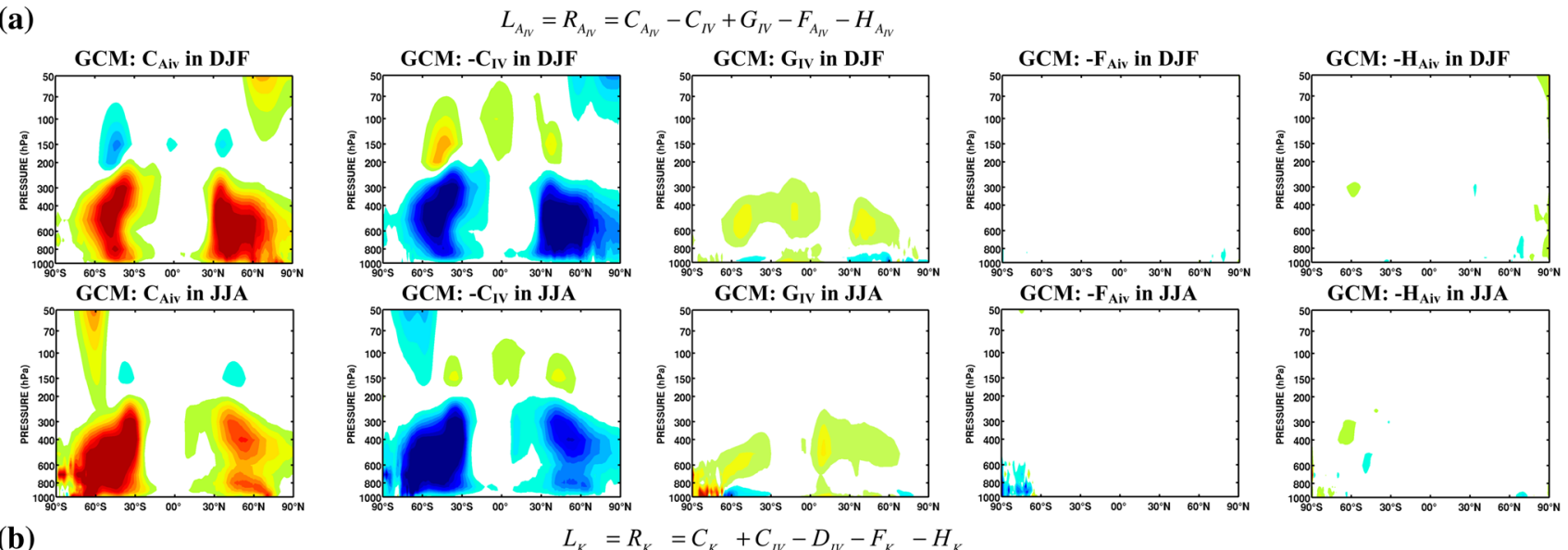

(b)
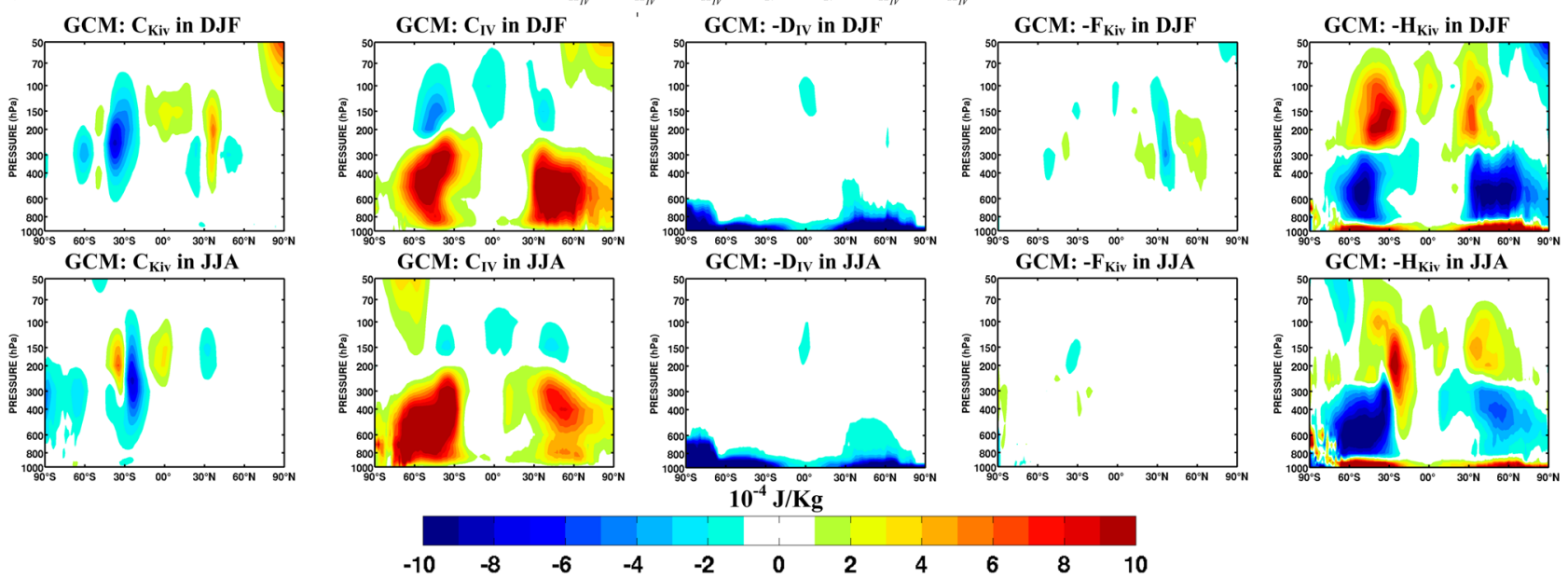

Fig. 11 Pressure-latitude cross-sections of the zonally averaged contributions to the GCM inter-member variability available enthalpy ( $\left.\mathrm{A}_{\mathrm{IV}}\right)$ tendency $\left(R_{A_{I V}}\right.$, upper two rows-a) and kinetic energy $\left(\mathrm{K}_{\mathrm{IV}}\right)$ tendency $\left(R_{K_{I V}}\right.$, bottom two rows- $\left.\mathbf{b}\right)$, in DJF and JJA

characteristic of being mainly positive over the continent and negative over the ocean in winter.

It was noted earlier that the boundary-flux term $-F_{A_{T V}}$ and the third-order term $-H_{A_{T V}}$ have weak magnitudes when averaged over the domain (Fig. 12); these fields however exhibit locally non-negligible values in the three datasets. In the GCM these terms are also similar to the patterns of $-H_{A_{I V}}$ and $-F_{A_{I V}}$. In the RCM, the terms $-H_{A_{I V}}$ and $-F_{A_{I V}}$ are rather negligible, but we note that $-F_{A_{I V}}$ reflects the fact that $A_{I V}$ energy is transported out of the regional domain by the mean flow.

The three datasets exhibit similar horizontal pattern of $-H_{K_{T V}}$, with dominant negative values resulting from values in the middle troposphere (from 250 to $850 \mathrm{hPa}$ ), only partly offset by values of opposite sign below $850 \mathrm{hPa}$, as seen in Fig. 14. Similar results are also seen for $-H_{K_{I V}}$ in the GCM, while RCM values are much smaller and exhibit different pattern.

The last row in Figure $\mathrm{S} 2$ displays the contribution $-F_{K_{T V}}$ for the three datasets, and $-F_{K_{I V}}$ for the two climate models.
All the maps show mainly negative values mostly over the eastern part of Canada, indicating that $K_{T V}$ and $K_{I V}$ loose energy by their transport outside this specific regional domain.

\section{Discussion and conclusion}

We compared the energetics of Available Enthalpy (AE) and Kinetic Energy (KE) transient-eddy variability (TV) and inter-member variability (IV) from two ensembles of simulations, one from a Global Climate Model (GCM) and one from a Regional Climate Model (RCM) integrated over an eastern North American domain. The models' TV statistics were compared to those of the Era-Interim reanalysis, considered here as the reference. The 30 GCM members and 50 RCM members simulations were initialised from the reanalysis, starting on different dates shifted by $24 \mathrm{~h}$ from 12 October 2004 at 0000 UTC, and were run till the 31 December 2005, using the sea-surface conditions from the 
(a)

DJF

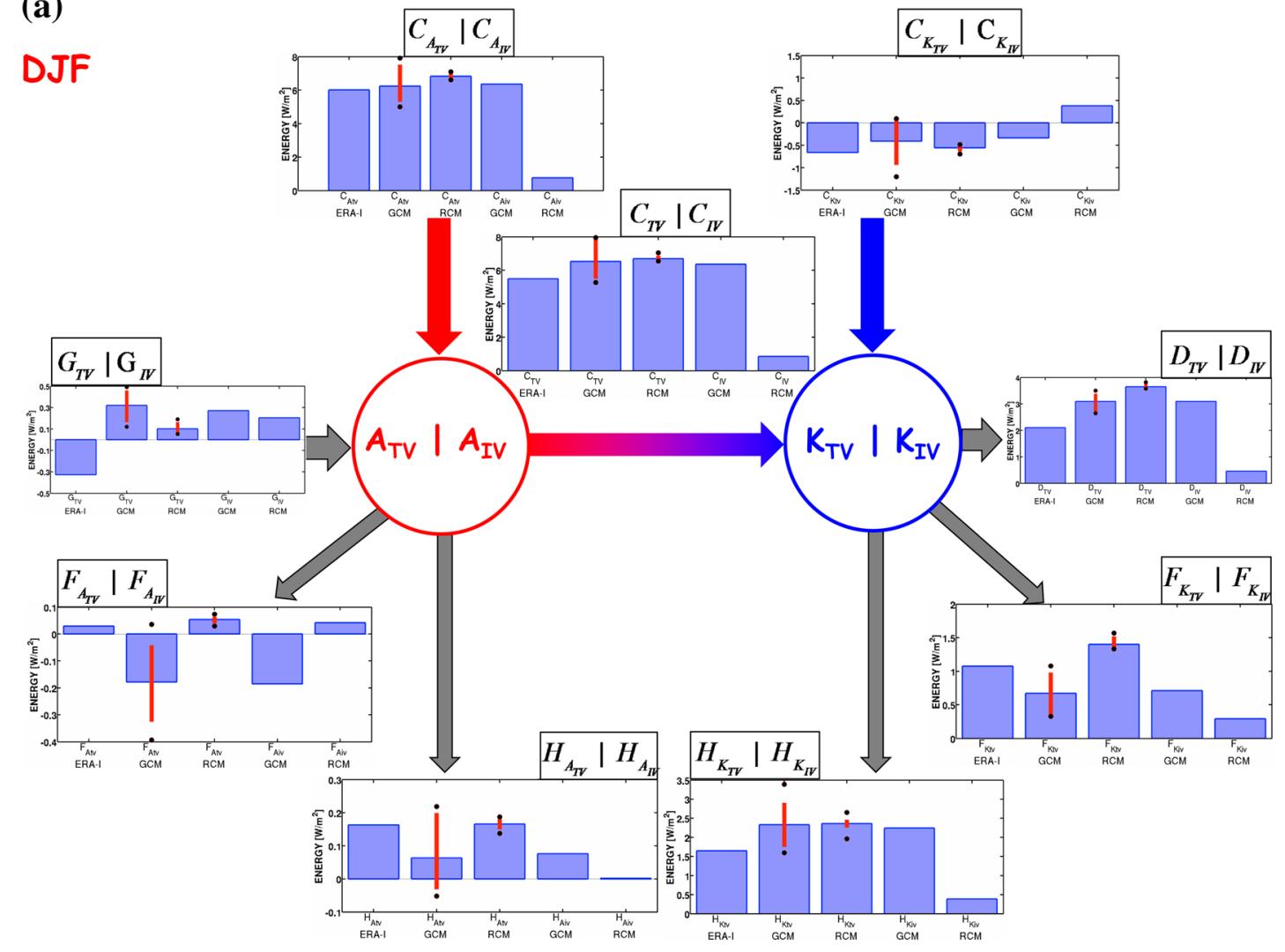

$C_{K_{T V}} \mid \mathrm{C}_{K_{I V}}$

(b)

DJF

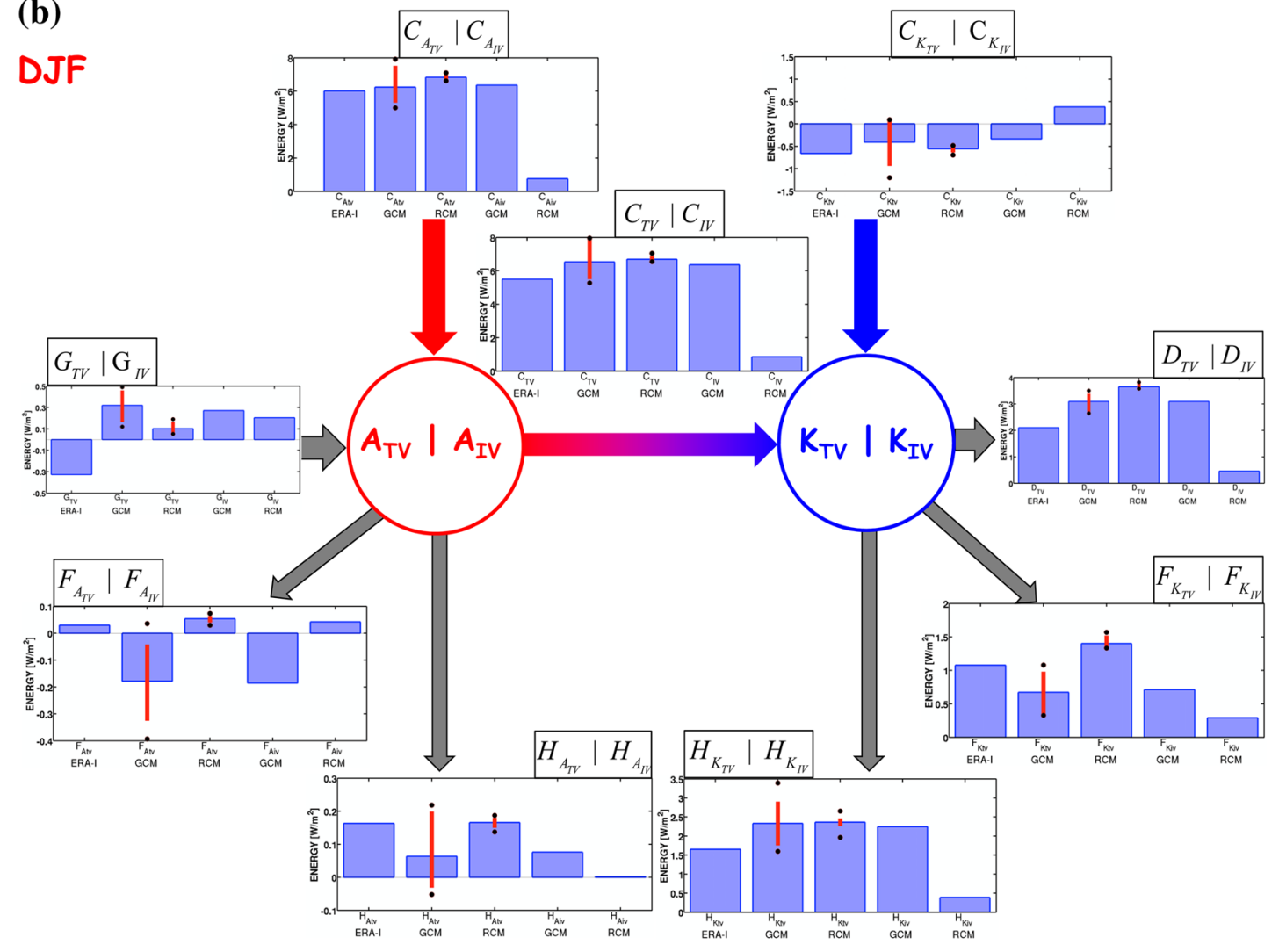

$C_{K_{T V}} \mid \mathrm{C}_{K_{I V}}$ 
4 Fig. 12 Energy cycles associated with transient-eddy $\left(\mathrm{A}_{\mathrm{TV}}\right.$ and $\mathrm{K}_{\mathrm{TV}}$, respectively) and inter-member variability $\left(\mathrm{A}_{\mathrm{IV}}\right.$ and $\mathrm{K}_{\mathrm{IV}}$, respectively), for reanalysis, GCM and RCM simulations, in DJF (a) and JJA (b), averaged over the regional domain shown as the blue rectangle in Fig. 1c. For GCM and RCM, the ensemble-mean values of contributions in TV energy budgets are shown; the red bar shows the $5-95 \%$ percentiles range around the mean, and the red dots represent the minimum and maximum values in the ensemble

reanalysis. All the members in the RCM simulations used the same lateral boundary conditions (LBC) from reanalyses, which limits IV due to the control exerted by the LBC in nested models. The study focussed on the two solstice seasons, December 2004-January 2005-February 2005 (noted DJF) and June-July-August of 2005 (noted JJA).

In the reanalysis, transient-eddy Available Enthalpy and Kinetic Energy ( $A_{T V}$ and $K_{T V}$, respectively) are found mostly near the storm track over mid-latitudes in both hemispheres. The maximum values of $A_{T V}$ are especially intense over North America in winter, indicating that this region is subject to large variations of temperature. The vertical distribution of the zonally averaged $K_{T V}$ shows that the maximum is associated with wind fluctuations in the jet stream near the tropopause in mid-latitudes. The GCM transient-eddy statistics are similar to those of the reanalysis; the spread of the transient-eddy statistics in the 30-member GCM ensemble is quite large, and in most cases encompasses the reanalysis values, confirming the skill of the GCM. Over the North American regional domain, the RCM's transient-eddy statistics are very similar to the reanalysis ones (in fact closer than the GCM), and the spread of the transient-eddy statistics in the 50-member RCM ensemble is much smaller than that in the GCM ensemble, owing to the control exerted upon the RCM simulations by the LBC provided by the reanalysis.

In the GCM simulations, the statistics of inter-member variability are very similar to those of transient-eddy variability, in agreement with the ergodicity property. The situation is quite different for the RCM simulations where the inter-member variability is much lesser and vanishes near the perimeter of the regional domain due to the control exerted by LBC that are identical for all members in the ensemble. Over North America the GCM's inter-member variability undergoes an annual cycle that reflects the amplitude of transient eddies, with maximum/minimum intensity occurring in winter/summer. The RCM's inter-member variability however has much weaker amplitude and it exhibits brisk fluctuations, with episodes of large growth followed by rapid decay within a few days. Furthermore, large values of IV energies are found in summer for RCM because of

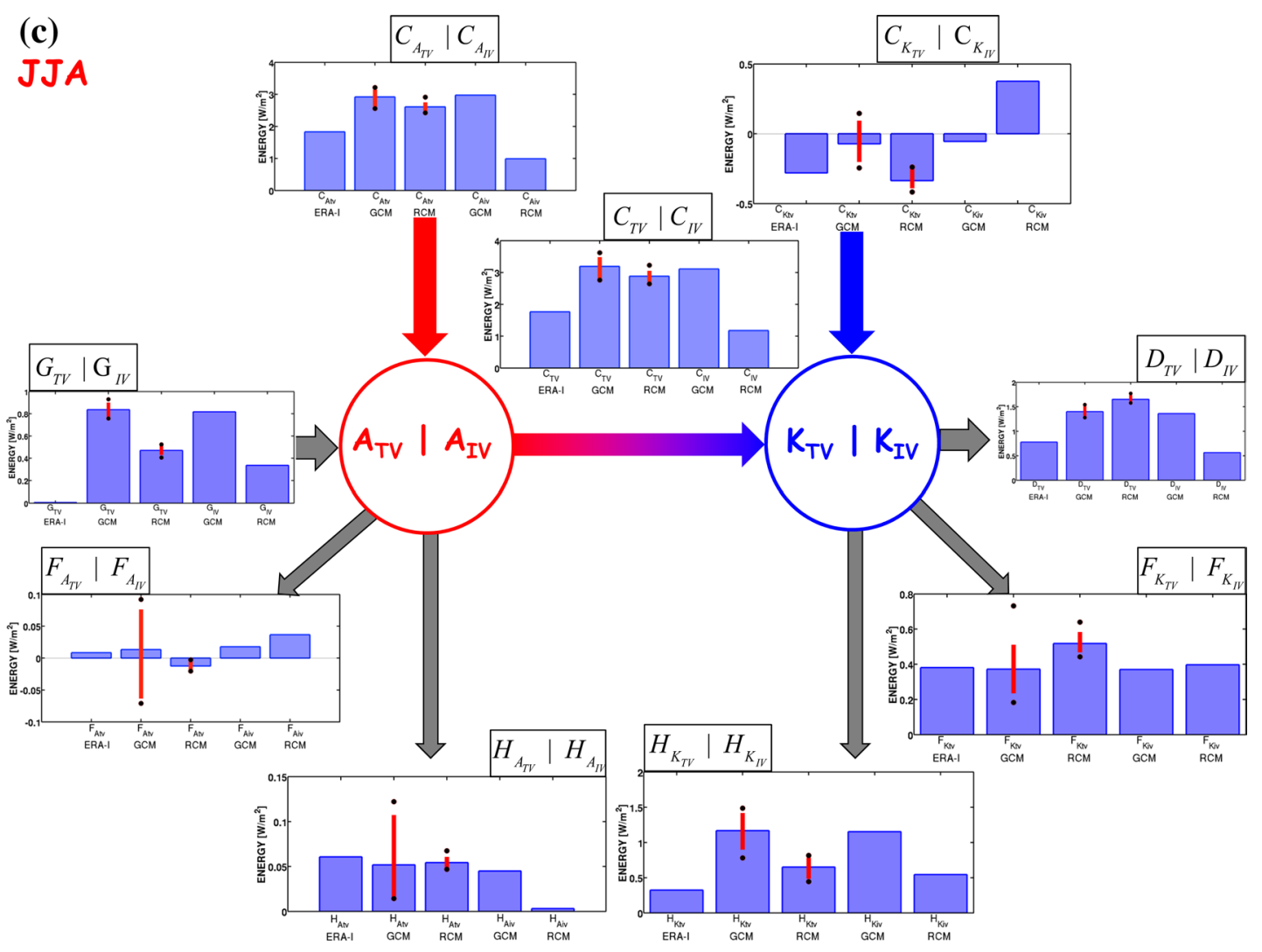

Fig. 12 (continued) 


$$
L_{A_{T V}}=C_{A_{T V}}-C_{T V}+G_{T V}-F_{A_{T V}}-H_{A_{T V}} \text { and } L_{A_{I V}}=C_{A_{I V}}-C_{I V}+G_{I V}-F_{A_{I V}}-H_{A_{I V}}
$$
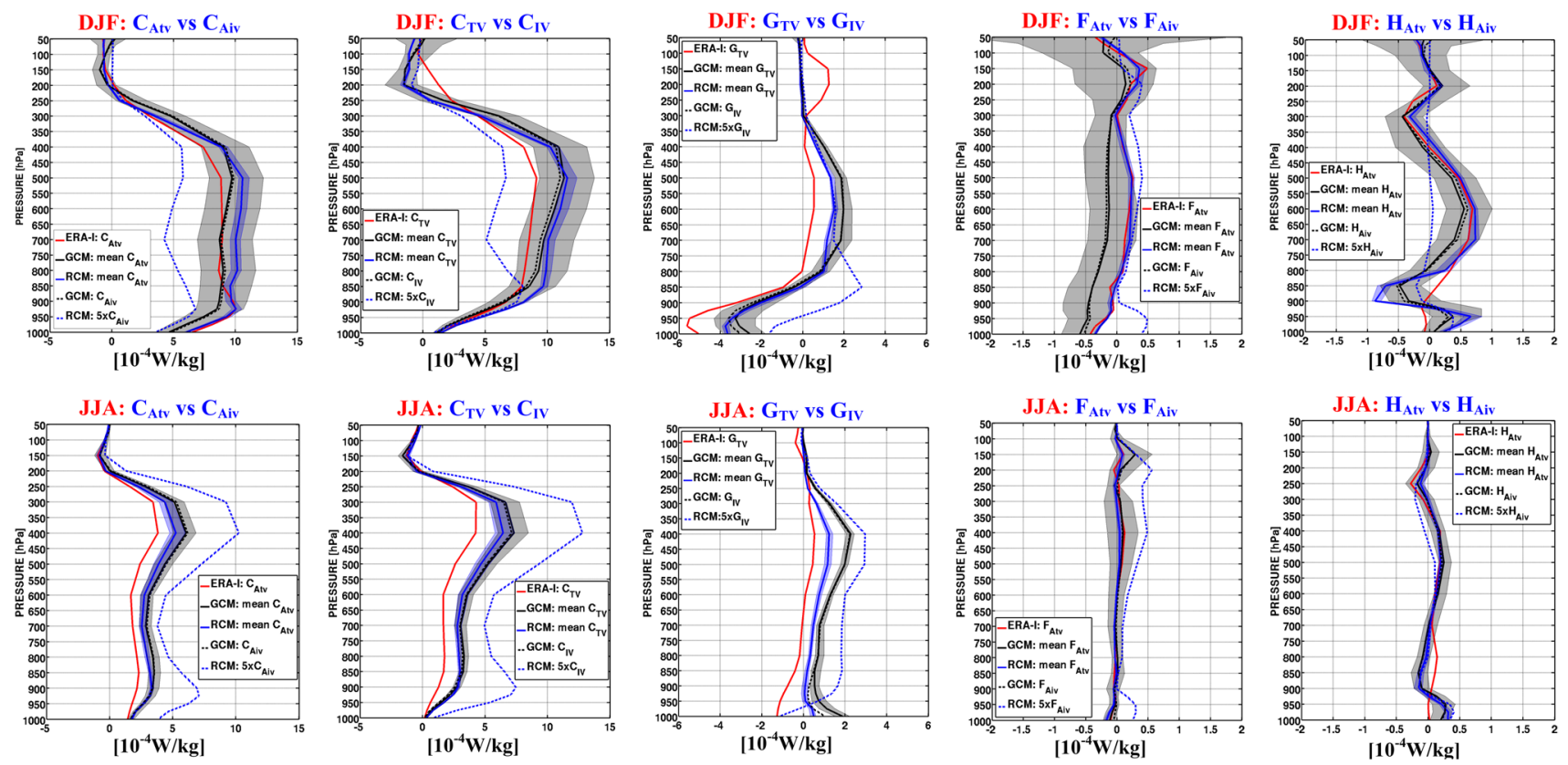

Fig. 13 Vertical profiles of horizontally averaged contributions in the budget equations of transient-eddy and inter-member variability available enthalpy $\left(\mathrm{A}_{\mathrm{TV}}\right.$ and $\mathrm{A}_{\mathrm{IV}}$, respectively), for reanalysis, GCM and RCM simulations, in DJF (first row) and JJA (second row). The gray and blue light bands represent the ranges of values for terms associ- ated with TV energy tendencies for the GCM and RCM, respectively, and their ensemble-mean profiles are shown in black and blue lines, respectively. The profiles of terms in $\mathrm{A}_{\mathrm{TV}}$ budget for reanalysis are presented in red lines. The profiles of $A_{I V}$ budget for the GCM and RCM are shown in black and blue dashed lines, respectively
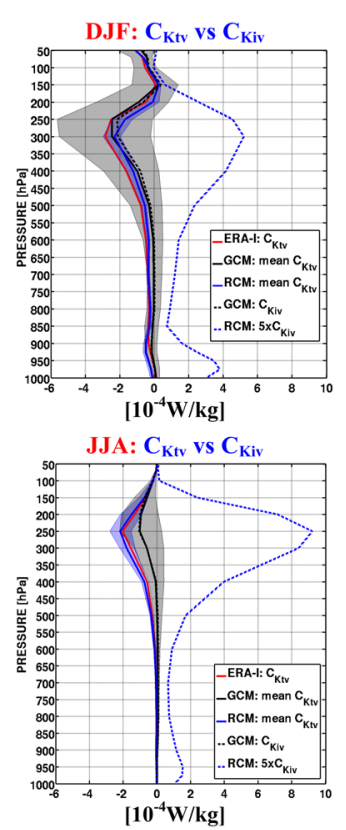
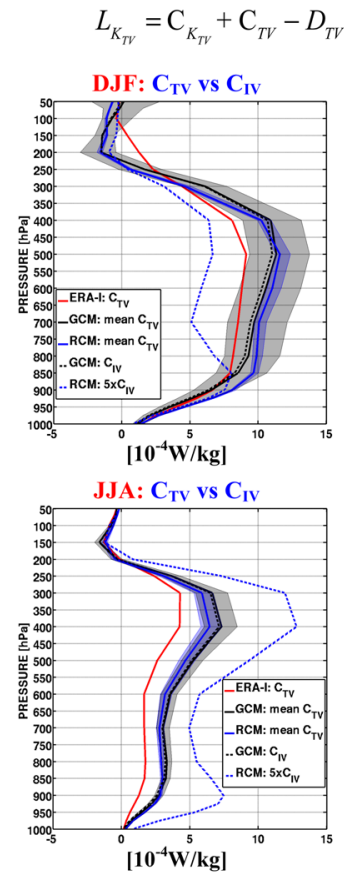
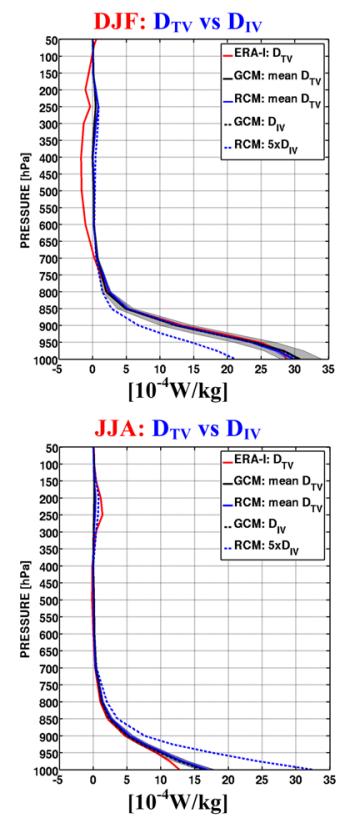
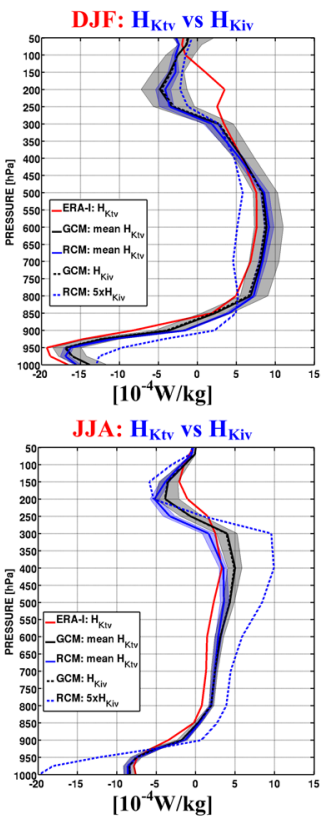

Fig. 14 As in Fig. 13, but for kinetic energy variabilities, $K_{T V}$ and $K_{I V}$ 
stronger local processes, such as condensation and convection, while in winter the stronger advection of IV energy out of the domain lead to a weak IV energy.

This study also analysed the energetics of AE and KE reservoirs associated with transient-eddy variability $\left(A_{T V}\right.$ and $\left.K_{T V}\right)$ and inter-member variability $\left(A_{I V}\right.$ and $\left.K_{I V}\right)$. Together these two energies (AE and KE) constitute a cycle with conversion of energy from one to the other. At the global scale, transient-eddy energetics budgets from reanalysis and GCM are quite similar and, in the GCM, the inter-member variability energetics are quite similar to those of transient eddies. The results indicate that the most important perturbation energy exchanges operate over the mid-latitudes in the troposphere.

Over the North American domain, the energetics of the GCM simulations and reanalysis are quite similar, as well as RCM's simulation TV energetics. Energy is supplied to the transient-eddy available enthalpy $\left(A_{T V}\right)$ reservoir mainly by the term $C_{A_{T V}}$ that converts available enthalpy from its timemean state to transient eddies. This conversion term is maximum in mid-latitudes where weather systems transport heat poleward through covariance of temperature and wind perturbations $\left(\overrightarrow{V^{\prime \prime}} T^{\prime \prime}\right)$ down-the-gradient of time-mean temperature field $(\vec{\nabla} \bar{T})$. The term $C_{T V}$ transfers energy from transient-eddy available enthalpy $\left(A_{T V}\right)$ to transient-eddy kinetic energy $\left(K_{T V}\right)$ through baroclinic conversion, with warm/cold air rising/sinking on average in mid-latitude weather systems, due to covariance of temperature and vertical motion perturbations $\left(\overline{\omega^{\prime \prime} \alpha^{\prime \prime}}\right)$. Transient-eddy kinetic energy is lost mainly through two physical processes: dissipation $\left(D_{T V}\right)$ and transport outside the regional domain $\left(F_{K_{T V}}\right)$. In the planetary boundary layer where the dissipation processes are important, results indicate that the term $D_{T V}$ is partly offset by the vertical component of the boundary term $-H_{K_{I V}}$ because of Ekman pumping $\left(-\frac{\partial}{\partial p} \overline{\Phi^{\prime \prime} \omega^{\prime \prime}}>0\right)$. For example,

in a low-pressure system $\left(\Phi^{\prime \prime}<0\right)$, warm air near the surface is forced to rise due to friction-induced convergence $\left(\omega^{\prime \prime}<0\right)$, leading to a positive covariance of fluctuations $\left(\overline{\Phi^{\prime \prime} \omega^{\prime \prime}}>0\right)$; negative vertical variation of the covariance $\left(\frac{\partial}{\partial p} \overline{\Phi^{\prime \prime} \omega^{\prime \prime}}<0\right)$ follows because covariance increases with height in low levels.

The analysis of the GCM simulations ensemble reveals that the energetics of inter-member variability is very similar to that of transient eddies, again confirming the ergodicity property of GCM ensembles. It is most interesting that IV and TV energy cycles share the same physical interpretation, with for example "baroclinic" conversion $C_{I V}$ occurring for notional inter-member perturbations just as it does through $C_{T V}$ for actual transient eddies in weather systems. The RCM simulations ensemble on the other hand exhibits much weaker inter-member variability energy and conversion intensity compared to those of GCM, which makes sense due to the control exerted by LBC that are identical for all members in the RCM ensemble. It is noteworthy that IV conversions occur in the RCM ensemble similar to those in the GCM ensemble, but with reduced amplitude, leading to similar physical interpretations for most of the terms in the budget; one main difference is the importance of the inter-member variability export out of the RCM domain. Despite the fact that inter-member variability is often considered a source of uncertainty for climate models, the present study confirms that it arises from the chaotic nature of the atmosphere and that it is not associated with numerical artefact, in particular, in the case of nested models.

Acknowledgements This research was co-funded by the Climate Change and Atmospheric Research (CCAR) programme of the Natural Sciences and Engineering Research Council of Canada (NSERC) through a grant to the "Canadian Network for Regional Climate and Weather Processes" (CNRCWP; http://www.cnrcwp.uqam.ca), by the project "Marine Environmental Observation, Prediction and Response" (MEOPAR; http://meopar.ca) of the Canadian Networks of Centres of Excellence, and by the NSERC Discovery Accelerator Supplements Program. The calculations were made on the Guillimin supercomputer of Compute Canada - Calcul Québec whose operation is funded by the Canada Foundation for Innovation (CFI), NanoQuébec, RMGA and the Fonds de recherche du Québec_-Nature et technologies (FRQ-NT). The authors thank Mr. Georges Huard and Mrs. Nadjet Labassi and Mrs. Katja Winger for maintaining an efficient and user-friendly local computing facility.

Open Access This article is distributed under the terms of the Creative Commons Attribution 4.0 International License (http://creativecommons.org/licenses/by/4.0/), which permits unrestricted use, distribution, and reproduction in any medium, provided you give appropriate credit to the original author(s) and the source, provide a link to the Creative Commons license, and indicate if changes were made.

\section{References}

Alexandru A, de Elia R, Laprise R (2007) Internal Variability in regional climate downscaling at the seasonal scale. Mon Weather Rev 135:3221-3238

Bélair S, Mailhot J, Girard C, Vaillancourt P (2005) Boundary-layer and shallow cumulus clouds in a medium-range forecast of a large-scale weather system. Mon Weather Rev 133:1938-1960

Bélair S, Roch M, Leduc AM, Vaillancourt PA, Laroche S, Mailhot J (2009) Medium-range quantitative precipitation forecasts from Canada's new 33-km deterministic global operational system. Weather Forecast 24:690-708. doi:10.1175/2008WAF2222175.1

Boer GJ (1974) Zonal and eddy forms of the available potential energy equations in pressure coordinates. Tellus 27(5):433-442

Caya D, Biner S (2004) Internal variability of RCM simulations over an annual cycle. Clim Dyn 22:33-46

Christensen OB, Gaertner MA, Prego JA, Polcher J (2001) Internal variability of regional climate models. Clim Dyn 17:875-887

Clément M, Nikiéma O, Laprise R (2016) Limited-area atmospheric energetics: illustration on a simulation of the CRCM5 over eastern North America for December 2004. Clim Dyn. doi:10.1007/ s00382-016-3198-0 
de Elía R, Caya D, Côté H, Frigon A, Biner S, Giguère M, Paquin D, Harvey R, Plummer D (2008) Evaluation of uncertainties in the CRCM-simulated North American climate. Clim Dyn 30:113132. doi:10.1007/s00382-007-0288-z

Dee DP, Uppala SM, Simmons AJ, Berrisford P, Poli P, Kobayashi S, Andrae U, Balmaseda MA, Balsamo G, Bauer P (2011) The ERA-Interim reanalysis: configuration and performance of the data assimilation system. Q J R Meteorol Soc 137:553-597. doi:10.1002/qj.828

Giorgi F, Bi X (2000) A study of internal variability of regional climate model. J Geophys Res 105:29503-29521

Girard C, Plante A, Desgagné M, McTaggart-Cowan R, Côté J, Charron M, Gravel S, Lee V, Patoine A, Qaddouri A, Roch M, Spacek L, Tanguay M, Vaillancourt PA, Zadra A (2014) Staggered vertical discretization of the Canadian environmental multiscale (GEM) model using a coordinate of the log-hydrostaticpressure type. Mon Weather Rev 142:1183-1196. doi:10.1175/ MWR-D-13-00255.1

Hernández-Díaz L, Laprise R, Sushama L, Martynov A, Winger K, Dugas B (2013) Climate simulation over CORDEX Africa domain using the fifth-generation Canadian regional climate model (CRCM5). Clim Dyn 40(5-6):1415-1433. doi:10.1007/ s00382-012-1387-z

Lorenz EN (1955) Available potential energy and the maintenance of the general circulation. Tellus 7:157-167

Lorenz EN (1963) Deterministic non-periodic flow. J Atmos Sci 42:433-471

Lorenz EN (1967) The nature and theory of the general circulation of the atmosphere. WorldMeteorol Organ 218 TP 115:161

Lucas-Picher P, Caya D, Biner S (2004) RCM's internal variability as function of domain size. Research activities in atmospheric and oceanic modelling, WMO/TD. J Côté Ed 1220 34:7.27-7.28

Lucas-Picher P, Caya D, de Elía R, Laprise R (2008) Investigation of regional climate models'internal variability with a ten-member ensemble of 10-year simulations over a large domain. Clim Dyn 31:927-940. doi:10.1007/s00382-008-0384-8

Marquet P (1991) On the concept of energy and available enthalpy: application to atmospheric energetics. Q J R Meteorol Soc 117:449-475

Marquet P (2003a) The available-enthalpy cycle. I: Introduction and basic equations. Q J R Meteorol Soc 129(593):2445-2466

Marquet P (2003b) The available-enthalpy cycle. II: Applications to idealized baroclinic waves. Q J R Meteorol Soc 129(593):2467-2494
McTaggart-Cowan R, Girard C, Plante A, Desgagné M (2011) The utility of upper-boundary nesting in NWP. Mon Weather Rev 139:2117-2144. doi:10.1175/2010MWR3633.1

Newell RE, Kidson JW, Vincent DG, Boer GJ (1972) The general circulation of the tropical atmosphere and interactions with extratropical latitudes. MIT Press, vol 1, p. 320

Newell RE, Kidson JW, Vincent DG, Boer GJ (1974) The general circulationof the tropical atmosphere and interactions with extratropical latitudes. MIT Press, vol 2, p 370

Nikiéma O, Laprise R (2011a) Diagnostic budget study of the internal variability in ensemble simulations of the Canadian RCM. Clim Dyn 36(11):2313-2337. doi:10.1007/s00382-010-0834-y

Nikiéma O, Laprise R (2011b) Budget study of the internal variability in ensemble simulations of the Canadian RCM at the seasonal scale. J Geophys Res Atmos. doi:10.1029/2011JD015841

Nikiéma O, Laprise R (2013) An approximate energy cycle for inter-member variability in ensemble simulations of a regional climate model. Clim Dyn 44(3-4):831-852. doi:10.1007/ s00382-012-1575-x

Nikiéma O, Laprise R (2015) Energy cycle associated with inter-member variability in a large ensemble of simulations with the Canadian RCM (CRCM5). Clim Dyn. doi:10.1007/s00382-015-2604-3

Oort AH (1964a) On estimates of the atmospheric energy cycle. Mon Weather Rev 92:483-493

Oort AH (1964b) On the energetics of the mean and eddy circulations in the lower stratosphere. Tellus 26(3):309-327

Pearce RP (1978) On the concept of available potential energy. Q J R Meteorol Soc 104:737-755

Rinke A, Dethloff K (2000) On the sensitivity of a regional Arctic climate model to initial and boundary conditions. Clim Res 14:101-113

Rinke A, Marbaix P, Dethloff K (2004) Internal variability in Arctic regional climate simulations: case study for the SHEBA year. Clim Res 27:197-209

van Mieghem J (1973) Atmospheric energetics. Oxford Univ Press

Verseghy LD (2000) The Canadian land surface scheme (CLASS): its history and future. Atmos Ocean 38:1-13

Verseghy LD (2008) The Canadian land surface scheme: technical documentation-version 3.4. ClimateResearch Division, Science and Technology Branch, Environment Canada

Weisse R, Heyen H, von Storch H (2000) Sensitivity of a regional atmospheric model to a sea state dependent roughness and the need of ensemble calculations. Mon Weather Rev 128:3631-3642 